\title{
Flare activity and photospheric analysis of Proxima Centauri ${ }^{\star}$
}

\author{
Y. Pavlenko ${ }^{1,2,3}$, A. Suárez Mascareño ${ }^{2,4,6}$, R. Rebolo ${ }^{2,4,5}$, N. Lodieu ${ }^{2,4}$, \\ V. J. S. Béjar ${ }^{2,4}$, and J. I. González Hernández ${ }^{2,4}$
}

\author{
${ }^{1}$ Main Astronomical Observatory of the National Academy of Sciences, 27 Zabolotnoho, Kyiv, Ukraine \\ e-mail: yp@mao.kiev.ua \\ 2 Instituto de Astrofísica de Canarias (IAC), Calle Vía Láctea s/n, 38200 La Laguna, Tenerife, Spain \\ 3 Center for Astrophysics Research, University of Hertfordshire, College Lane, Hatfield, Hertfordshire AL10 9AB, UK \\ ${ }^{4}$ Departamento de Astrofísica, Universidad de La Laguna (ULL), 38205 La Laguna, Tenerife, Spain \\ 5 Consejo Superior de Investigaciones Científicas, CSIC, Spain \\ ${ }^{6}$ Observatoire Astronomique de l'Université de Genève, 1290 Versoix, Switzerland
}

Received 6 March 2017 / Accepted 12 June 2017

\begin{abstract}
Context. We present the analysis of emission lines in high-resolution optical spectra of the planet-host star Proxima Centauri (Proxima) classified as a M5.5V.

Aims. We carry out a detailed analysis of the observed spectra to get a better understanding of the physical conditions of the atmosphere of this star.

Methods. We identify the emission lines in a series of 147 high-resolution optical spectra of the star at different levels of activity and compare them with the synthetic spectra computed over a wide spectral range.

Results. Our synthetic spectra computed with the PHOENIX 2900/5.0/0.0 model atmosphere fits the observed spectral energy distribution from optical to near-infrared quite well. However, modelling strong atomic lines in the blue spectrum (3900-4200 ̊̊) requires implementing additional opacity. We show that high-temperature layers in Proxima Centauri consist of at least three emitting parts: a) a stellar chromosphere where numerous emission lines form; we suggest that some emission cores of strong absorption lines of metals form there; b) flare regions above the chromosphere, where hydrogen Balmer lines up to high transition levels (10-2) form; and c) a stellar wind component with $V_{r}=-30 \mathrm{~km} \mathrm{~s}^{-1}$ seen in some Balmer lines as blueshifted emission lines. We believe that the observed He line at $4026 \AA$ in emission can be formed in that very hot region.

Conclusions. We show that the real structure of the atmosphere of Proxima is rather complicated. The photosphere of the star is best fit by a normal M5 dwarf spectrum. On the other hand, emission lines form in the chromosphere, flare regions, and extended hot envelope.
\end{abstract}

Key words. stars: individual: Proxima Cen - stars: atmospheres - stars: abundances - stars: activity - stars: flare - stars: fundamental parameters

\section{Introduction}

$\mathrm{M}$ dwarfs are the most numerous and longest-lived stars in our Milky Way (see Kirkpatrick et al. 2012). Unfortunately, the determination of the basic parameters of these stars is hampered by the complicated physical processes taking place in their atmospheres which limit our ability to reproduce their spectra with synthetic models. Owing to the low temperatures and high pressures in $\mathrm{M}$ dwarf photospheres, modelling their spectra requires detailed accounting for molecules when dealing with chemical equilibrium in their atmospheres. $\mathrm{M}$ dwarf spectra are governed by absorptions of the numerous band systems of diatomic and polyatomic molecules. Spectra of M dwarfs also show emission lines which can be formed only in the outermost hightemperature layers of their atmospheres.

Proxima Centauri (=2MASS J14294291-6240465; GJ 551, V645 Cen) is the closest red dwarf to the Sun; it is located at a distance of $1.3019 \pm 0.0018 \mathrm{pc}$ (Lurie et al. 2014). Because of its proximity, its angular radius can be measured directly via

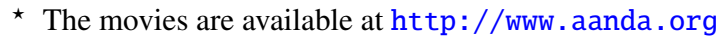

interferometry (Kervella et al. 2003). Its mass is about an eighth of the Sun's mass, its luminosity is only $0.15 \%$ of that emitted by the Sun, its spectral type is M5.5 (Bessell 1991), its effective temperature is $3050 \mathrm{~K}$, and its density is about 40 times that of our Sun. Since its discovery, Proxima Centauri has been thought to be the third component of the $\alpha$ Centauri system. Recently, Kervella et al. (2017), based on new observations, have claimed that Proxima and $\alpha$ Cen are gravitationally bound with a high degree of confidence. It may be the third component of the $\alpha$ Centauri system with a projected physical separation of $15000 \pm 700$ au (Wertheimer \& Laughlin 2006).

Proxima Cen is a known flare star that exhibits random but significant increases in brightness due to magnetic activity (Christian et al. 2004). The spectrum of Proxima Cen contains numerous emission lines (see Fuhrmeister et al. 2011). These features most likely originate from plages, spots, or a combination of both. In general, the flare rate of Proxima Cen is lower than that of other flare stars of similar spectral type, but is unusually high given its slow rotation period (Davenport et al. 2016). The star has an estimated rotation period 
of $\sim 83$ days and a magnetic cycle of $\sim 7 \mathrm{yr}$ (Benedict et al. 1998; Suárez Mascareño et al. 2015, 2016; Wargelin et al. 2017). The $\mathrm{X}$-ray coronal and chromospheric activity have been studied in detail by Fuhrmeister et al. (2011) and Wargelin et al. (2017). Recently, Thompson et al. (2017) have claimed detection of rotational modulation of atomic lines in the $\alpha$ Cen B spectrum.

Today, $\mathbf{M}$ dwarfs represent important targets for searches of exoplanets, and in particular rocky planets. Given their small radius and low mass, planets are easier to detect around $M$ dwarfs because the depth of their transits and the amplitudes of the induced radial velocity variations are larger. The first rocky planets were detected by radial velocity and transits around M stars (Rivera et al. 2015; Charbonneau et al. 2009). Most of the rocky planets in the habitable zone have been found around these very low-mass stars (Udry et al. 2007; Bonfils et al. 2011; Quintana \& Barclay 2014; Torres et al. 2015; Wright et al. 2016; Anglada-Escudé et al. 2016). Proxima Cen was recently highlighted as a planet-host mid-M dwarf (Anglada-Escudé et al. 2016). Proxima Cen b orbits its host star with a period of 11.2 days, corresponding to a semi-major axis distance of 0.05 AU. Proxima Cen b has a mass close to that of the Earth (from 1.10 to 1.46 mass of the Earth), and orbits in the temperate zone (Anglada-Escudé et al. 2016).

One may assume that Proxima Cen b is surrounded by an atmosphere with a surface pressure of one bar, implying that the planet orbits its host star within the habitable zone (Ribas et al. 2016; Turbet et al. 2016; Garraffo et al. 2016). It is worth noting that the classical definition of the habitable zone solely implies the restriction of distance from the central star and composition of the planetary atmosphere. However, strong flare activity may move the inner boundary of the habitable zone far away from the formally computed possible radius. For this reason, the detailed characterisation of the flare phenomenon present in the atmosphere of Proxima Cen is of great importance.

In this paper we report on the detection of emission lines in the optical spectra of Proxima taken with different instruments ran by the European Southern Observatory (ESO). In Sect. 2 we describe the observations and data reduction. In Sect. 3 we analyse the characteristics of several lines, including Balmer lines, sodium resonance doublets, and the $\mathrm{Ca}$ II $\mathrm{H}$ and $\mathrm{K}$ lines. In Sect. 4 we place our results into a wider context of activity in low-mass stars.

\section{Spectroscopic observations}

\subsection{6 m/HARPS optical spectra of Proxima}

We retrieved all the available Proxima spectra from the HARPS ESO public data archive. The dataset consists of 316 spectra collected between June 2004 and May 2016. HARPS (Mayor et al. 2003) is a fibre-fed high-resolution echelle spectrograph installed at the $3.6 \mathrm{~m}$ ESO telescope in La Silla Observatory (Chile). The instrument has a resolving power $R \sim 115000$ over a spectral range from 3780 to $6810 \AA$ and has been designed to attain very high long-term radial velocity (RV) accuracy. It is contained in a vacuum vessel to avoid spectral drifts due to temperature and air pressure variations, thus ensuring its stability. HARPS is equipped with its own pipeline providing extracted and wavelength-calibrated spectra, as well as RV measurements and other data products such as cross-correlation functions and their bisector profiles. In order to avoid contamination of the stellar spectra by the calibration lamp we relied only on those spectra taken without simultaneous calibration. The final selection consisted of 147 high-resolution spectra taken from the public
ESO archive, observed between 2004 and 2016 with exposure times ranging from 450 to $1200 \mathrm{~s}$.

For the analysis we used the reduced wavelength-calibrated spectra produced by the HARPS pipeline. We corrected every spectrum from the velocity of the star and created a high signalto-noise spectrum by co-adding all the available spectra.

\subsection{Spectra of Proxima in different states of activity}

We needed very high signal-to-noise spectra in order to perform a detailed study of the activity processes and spectral features of Proxima. To this end we created two high S/N spectra with two different sets of individual spectra. One set was created by co-adding all the available spectra, once set in the barycentric frame of reference and corrected for the radial velocity of the star, which gave us a final spectrum containing the information of the spectral features both in times of strong and weak activity of Proxima. We denote the resulting spectrum "S". Then we created a second spectrum for which we filtered out the spectra obtained during flares, and created a mean spectrum representing the times of quietness of the star by once again co-adding the selected spectra. We denote this spectrum "QC". Flares are identified by measuring unusually high levels of $\mathrm{Ca}$ II $\mathrm{H} \& \mathrm{~K}$ emission and $\mathrm{H}_{\alpha}$ emission. We measure the Mount Wilson $\mathrm{S}$ index (Noyes et al. 1984) and the $\mathrm{H}_{\alpha}$ index defined by Gomes da Silva et al. (2011) following the procedure illustrated in Suárez Mascareño et al. (2015). Spectra that show an S index exceeding the seasonal mean by more than 3 times the RMS of the whole series are considered in flare state. As a result of the process we obtain two spectra with $S / N>100$ in a wide spectral range in two different states (average state and low-activity state) which allows us to study the changes in its chromosphere related to changes in its activity level.

\subsection{VLT/X-shooter spectra}

$\mathrm{X}$-shooter is a multiwavelength cross-dispersed echelle spectrograph (D'Odorico et al. 2006; Vernet et al. 2011) mounted on the Cassegrain focus of the Very Large Telescope (VLT) Unit 2. The spectrograph is made of three arms covering simultaneously the ultraviolet (UVB; 3000-5500 ̊), visible (VIS; 5500-10 $000 \AA$ ), and near-infrared (NIR; 10000-24800 $\mathrm{A}$ ) wavelength ranges thanks to the presence of two diachronic splitting the light. The spectrograph is equipped with three detectors: a $4096 \times 2048 \mathrm{E} 2 \mathrm{~V}$ CCD44-82, a 4096 × 2048 MIT/LL CCID 20, and a 2096× 2096 Hawaii 2 RG for the UVB, VIS, and NIR arms, respectively.

We downloaded public data of Proxima from the European Southern Observatory (ESO) science archive. The VLT/Xshooter spectra were taken on 15 January 2014 between UT = $6 \mathrm{~h} 55$ and UT $=7 \mathrm{~h}$ as part of ESO program 092.D-0300. The observation strategy was $2 \mathrm{AB}$ cycles of $12 \mathrm{~s}, 33 \mathrm{~s}$, and $44 \mathrm{~s}$ in the UVB, VIS, and NIR arms, respectively. The slits of $0.5 \operatorname{arcsec}$ 0.4 arcsec, and 0.4 arcsec were used, yielding resolving powers of 9900 (3.2 pixels per full width half maximum), 18200 (92.9 pixels per full width half maximum), and 10500 (2.2 pixels per full width half maximum) in the UVB, VIS, and NIR arms, respectively. The read-out mode was set to $400 \mathrm{k}$ and low gain without binning.

We reduced the raw dataset with the latest version of the $\mathrm{X}$-shooter pipeline $(2.8 .0)^{1}$. The pipeline removes the instrumental signature to the raw spectra, including flat field and dark frame corrections. The spectra are wavelength-calibrated,

\footnotetext{
http://www. eso.org/sci/software/pipelines/
} 

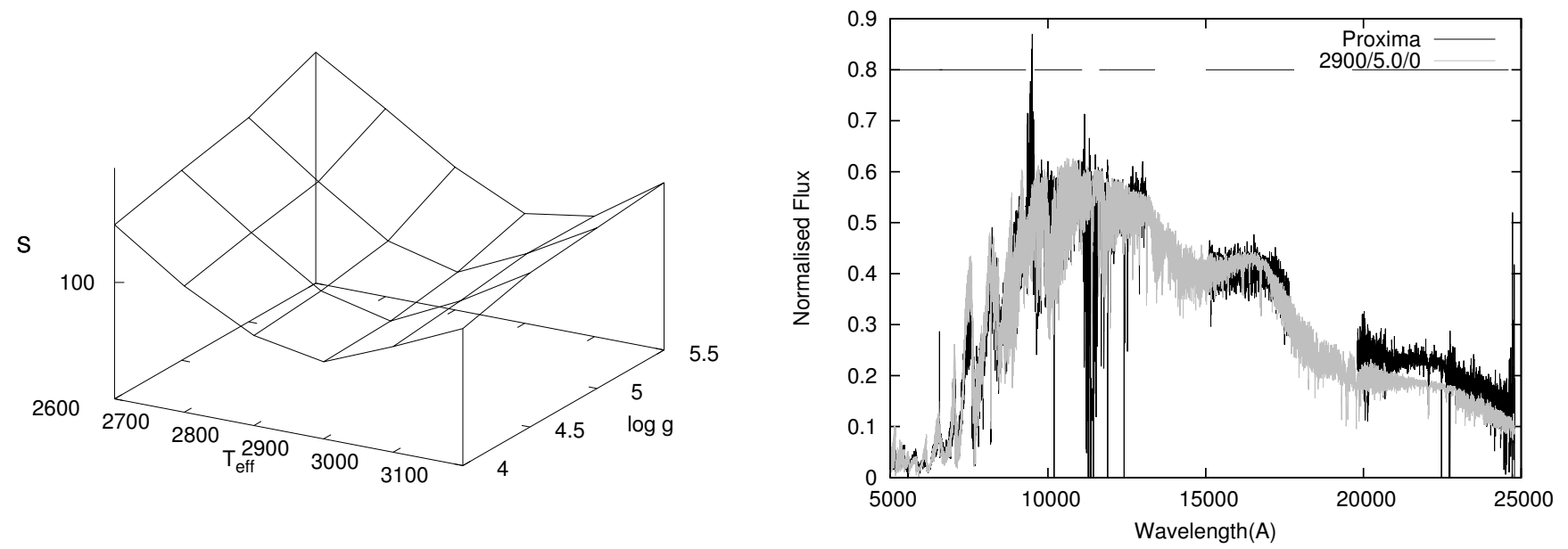

Fig. 1. Left: dependence of $S$ on $T_{\text {eff }}$, log g. Right: best fit of synthetic spectrum 2900/5.0/0 to the observed VLT/X-shooter spectrum of Proxima. We remove here the spectral regions of the strong telluric absorption in $J$ and $K$ bands; spectral ranges used for the fits are indicated by horizontal lines at $F_{\lambda}=0.8$.

sky-subtracted, and finally flux-calibrated with the associated spectro-photometric standard star observed as part of the ESO calibration plan. The output products include a $2 \mathrm{D}$ spectrum associated with a $1 \mathrm{D}$ spectrum. Nonetheless, we extracted the 1D UVB, VIS, and NIR spectra with the apsum task under IRAF ${ }^{2}$ (Tody 1986, 1993).

\section{Results}

\subsection{Absorption spectra of Proxima}

\subsubsection{Effective temperature from the X-shooter's SED}

Rajpurohit et al. (2013) and Passegger et al. (2016) showed that the effective temperature and gravity of normal field M5 dwarfs are $T_{\text {eff }}=2900 \pm 100 \mathrm{~K}$ and $\log g=5.0 \pm 0.5$, respectively. These values are most likely applicable to Proxima classified as a M5.5 dwarf with solar metallicity. This metallicity agrees well with the suggestion that Proxima is the third (C) component of the $\alpha$ Cen system (see Reipurth \& Mikkola 2012, and references therein).

We fitted our synthetic spectra computed with the Phoenix model atmospheres in the effective temperature $T_{\text {eff }}$ range [2600:3100 K] with incremental steps of $100 \mathrm{~K}$ and gravity $\log g$ [4.0:5.5] with steps of 0.5 dex to the observed VLT/Xshooter SED. In our work we adopted the solar abundances of Anders \& Grevesse (1989), except for iron abundance log $N(\mathrm{Fe})=-4.5$ in the scale $\sum N_{i}=1.0$. These abundances agree with Asplund et al. (2009) within accuracy $\sim 0.1$ dex for most elements. Nevertheless, our abundances allow to fit the spectra of the Sun and solar-like stars in good agreement with other authors, using comparative simple 1D model atmospheres (see Ivanyuk et al. 2017). The studies of Pavlenko (2014) and Pavlenko \& Schmidt (2015) provide reviews of the input data and a detailed explanation of the procedure employed to compute the synthetic spectra. Our least-squares fitting procedure is described in Pavlenko et al. (2006b). We chose the best fit of the computed spectra to the observed spectrum for the minimum of

2 IRAF is distributed by the National Optical Astronomy Observatories, which are operated by the Association of Universities for Research in Astronomy, Inc., under cooperative agreement with the National Science Foundation. the $S$ function defined as

$S\left(f_{\mathrm{h}}, \Delta \lambda, R\right)=\sum_{\lambda}\left(F_{\lambda}-f_{\mathrm{h}} * F_{\lambda}^{s}(\Delta \lambda, R)\right)^{2}$,

where $F_{\lambda}$ and $F_{\lambda}^{s}$ are the fluxes in the observed and computed fluxes, respectively, and $f_{\mathrm{h}}, \Delta \lambda, R$ are the normalisation flux factor, shift in wavelengths between observed and computed spectra, and the instrumental broadening factor $R$, respectively. We created fits for all synthetic spectra from our grid.

In the left panel of Fig. 1, we show the dependence of $S$ computed for the range of adopted parameters in effective temperature $T_{\text {eff }}$ and gravity $\log g$. We carried out our minimisation procedure only for the "good", i.e. without notable telluric absorption and/or emission features across $\lambda \lambda 6650$

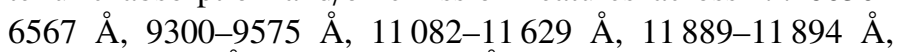
13 393-15000 $\mathrm{A}, 17808-19638 \AA$ (see Fig. 1). Shorter wavelengths of $\lambda<5000 \AA$ were excluded, due to some problems discussed in Sect. 3.1.5. We find a clear minimum of $S$ for $T_{\text {eff }}=2900 \mathrm{~K}$ for all considered cases of $\log g$. The dependence on $\log g$ is rather weak when varying the $T_{\text {eff }}$.

\subsubsection{Gravity from absorption lines in NIR VLT/X-shooter spectra of Proxima}

We showed that the fit to observed SED of Proxima suggests $T_{\text {eff }}=2900 \mathrm{~K}$ (Fig. 1). To constrain further the gravity of Proxima, we fit gravity-sensitive absorption lines present in the optical spectrum. Here we draw attention to the profiles of the atomic lines. It is worth noting that because the spectra of $\mathrm{M}$ dwarfs are complicated, the comparison of atomic line profiles in computed and observed spectra is not an easy task. The atomic lines in spectra of $\mathrm{M}$ dwarfs form at the background of the haze of molecular lines of different intensity. The molecular features/blends cannot be fitted as nicely as atomic lines. Nevertheless, comparison of observed and computed profiles of atomic lines allows us to constrain appropriate input parameters. In particular, here we focused our efforts on the resonance lines of potassium at 7664.9 and $7698.96 \AA$ and on the subordinate triplet of sodium at $8126 \AA$, which are well-known gravity indicators. We show the fits to these lines in Fig. A.1. We can conclude that the optical spectrum of Proxima is best reproduced with solar abundances and $\log g=5.0$ dex. Our results agrees within the 


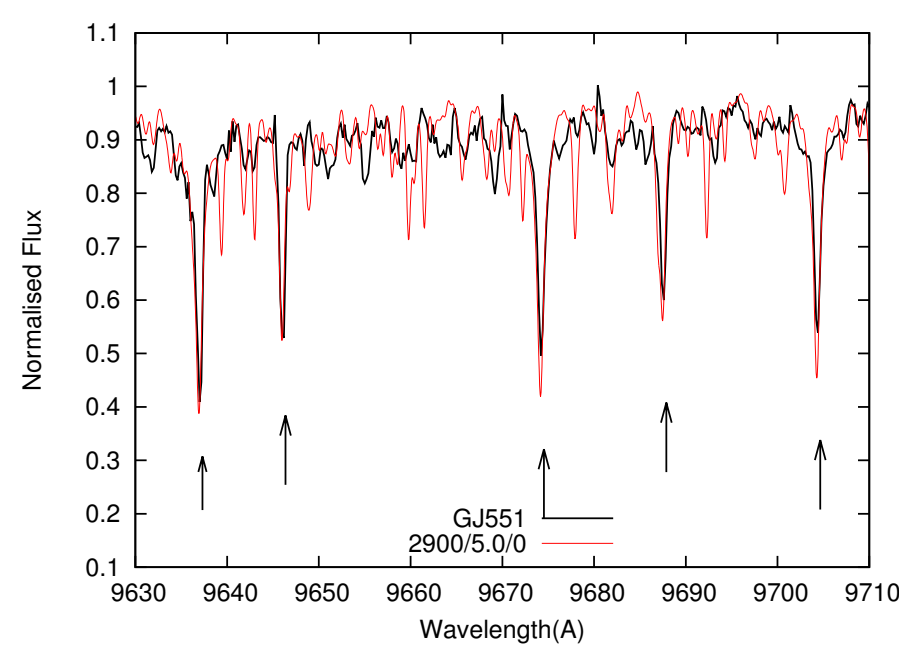

Fig. 2. Synthetic fit to Ti I lines marked by arrows in the observed VLT/X-shooter spectrum.

uncertainties with the $\log g=5.5$ dex derived by Passegger et al. (2016).

\subsubsection{CO bands}

In Fig. A. 2 we compare the observed spectrum of Proxima with the theoretical spectrum using the $\Delta v=2 \mathrm{CO}$ bands computed for the 2900/5.0/0 model atmosphere. We can see that the fit of the synthetic CO bands to the observed data is a good diagnostic to infer the physical conditions in $\mathrm{M}$ dwarf atmospheres (see Pavlenko \& Jones 2002). In particular we find that the synthetic spectra with the proper effective temperature match reasonably well the observations and agree with the temperatures derived by empirical methods. We see a rather marginal response to a presence of outer hot atmospheric layers, i.e. of a chromosphere, because $\mathrm{CO}$ is a very stable molecule of large dissociation potential $\left(D_{0}=11.105 \mathrm{eV}\right)$. In general, the $\mathrm{CO}$ bands are seen in absorption. At the resolution of our observations we can also conclude that our ${ }^{12} \mathrm{C} /{ }^{13} \mathrm{C}$ ratio is consistent with the solar because the ${ }^{13} \mathrm{CO}$ bands are weak or even absent in the observed spectrum (Fig. A.2).

\subsubsection{Abundances in the Proxima atmosphere}

In the previous sections we analysed the saturated lines of atoms and molecules, which by definition show a rather marginal dependence on the changes of abundances. We can assume here that the abundances of the alkali elements shown in Fig. A.1 do not differ much from the solar case. In the VLT/X-shooter spectra we also observe absorption lines of other elements at the background of the local pseudo-continuum formed by the molecular bands across the optical and NIR spectral ranges. Therefore, to make our abundance analysis more reliable, we employ a few atomic lines of intermediate strength present in the red part of the optical spectrum of Proxima where molecular absorption is weaker. We discuss these lines below.

Titanium. Lines of TiI are more numerous than FeI in the spectrum of Proxima, due to the lower potential of ionisation of titanium. In Fig. 2 we show the fit with the synthetic spectrum computed for the 2900/5.0 model atmosphere with the solar log $N(\mathrm{Ti})=-7.05$ (Grevesse \& Sauval 1998). In Table 1 we list the derived abundances. We find a Ti abundance of $\log N(\mathrm{Ti})=$ $-7.20 \pm 0.15$ from an average of five TiI lines, suggesting a weak
Table 1. Abundances of Til obtained from the fits to lines shown in Fig. 2.

\begin{tabular}{cccc}
\hline \hline$\lambda$ & $g f$ & $E^{\prime \prime}(\mathrm{eV})$ & $\log N(\mathrm{Ti})$ \\
\hline 9638.31 & $2.44 \mathrm{E}-01$ & 0.848 & $-7.05 \pm 0.15$ \\
9647.37 & $3.68 \mathrm{E}-02$ & 0.818 & $-7.05 \pm 0.15$ \\
9675.54 & $1.57 \mathrm{E}-01$ & 0.836 & $-7.35 \pm 0.15$ \\
9688.87 & $2.45 \mathrm{E}-02$ & 0.813 & $-7.35 \pm 0.15$ \\
9705.66 & $9.79 \mathrm{E}-02$ & 0.826 & $-7.20 \pm 0.15$ \\
\hline
\end{tabular}

metal deficient atmosphere for Proxima. However, we used this abundance estimate with caution because we see that most of the observed absorption lines are weaker than the lines in the theoretical spectrum computed at solar abundance.

Iron. Iron lines are numerous in the spectrum of Proxima but not as intense as the TiI lines. Although weak lines are more affected by the uncertainties, the theoretical and observed spectra agree qualitatively well as shown in the left panel of Fig. 3. In the right panel of Fig. 3 we display the fit to the observed profile of the intense FeI line at $8327.06 \AA(g f=0.02985)$, shown also in Fig. A.1. We obtained good quantitative agreement for $\log$ $N(\mathrm{Fe})=-4.4$ dex, similar to the solar iron abundance within \pm 0.2 dex.

Lithium. The absorption bands of the TiO molecule govern the spectra of $\mathrm{M}$ dwarfs around the Li resonance doublet at $6707.8 \AA$. The Li doublet is not seen in the spectrum of Proxima as expected for a fully convective old low-mass star. We compare the observed HARPS spectra and synthetic spectra computed with the 2900/5.0/0.0 model atmosphere and the line lists from Plez (1998) and Schwenke (1998). Generally speaking, Schwenke's TiO line list more closely reproduces the shape of the blends across the pectral range containing the Li doublet. However, in our case we can only place an upper limit to the lithium abundance in the atmosphere of Proxima at log $N(\mathrm{Li})=-12.04$ dex (right panel in Fig. 4).

\subsubsection{Atomic and molecular absorption spectra in the 3800-4200 Å region}

Absorption lines of neutral species in the blue spectra of $M$ dwarfs are expected to be much stronger than in the solar case, due to the lower temperatures and higher pressures in the regions of the absorption line formation. Moreover, we may expect resonance lines of neutral metals to appear, due to the changes of their ionisation equilibrium in this low temperature regime.

To verify our treatment of the pressure broadening we compute two spectral regions in the solar spectrum containing lines that are rather strong. We follow the procedure described in Pavlenko et al. (1995) in our computations. The profile of the absorption line is described by a Voigt function $H(a, v)$ and the damping broadening parameter $a$ changes with depth in stellar atmosphere. We computed the synthetic spectra with the VALD3 line list (Ryabchikova et al. 2015; Ryabchikova \& Pakhomov 2015) for the 5777/4.44/0 solar model atmosphere (Pavlenko 2003 ) with a micro-turbulent velocity of $V_{\mathrm{m}}=1 \mathrm{~km} \mathrm{~s}^{-1}$ and wavelength steps of $0.025 \AA$. In Fig. A.3 we observe a good agreement between the profiles of strong atomic lines in the observed spectrum of the Sun as a star and the computed spectrum (Fig. A.3).

We computed the synthetic spectrum of Proxima across the 3800-4200 $\AA$ wavelength range. Molecular absorption is weak 

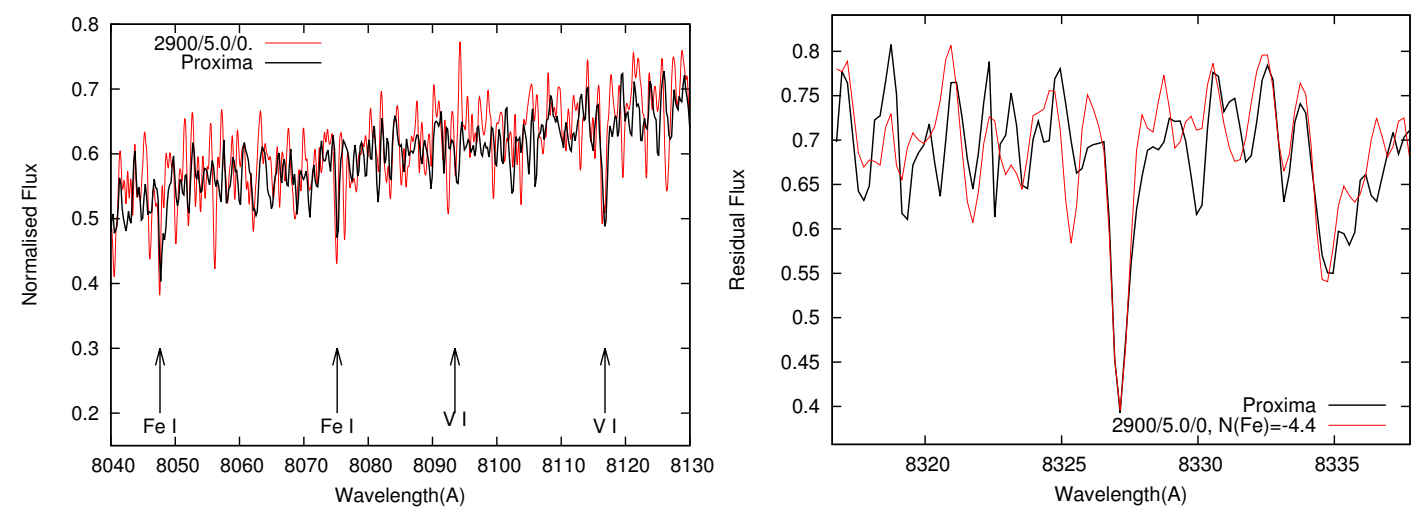

Fig. 3. Left: fits to the observed FeI and VI lines with the $2900 / 5.0 / 0$ model atmosphere. Right: fit to the observed FeI line at $8327.06 \AA$ with log $N(\mathrm{Fe})=-4.4$.
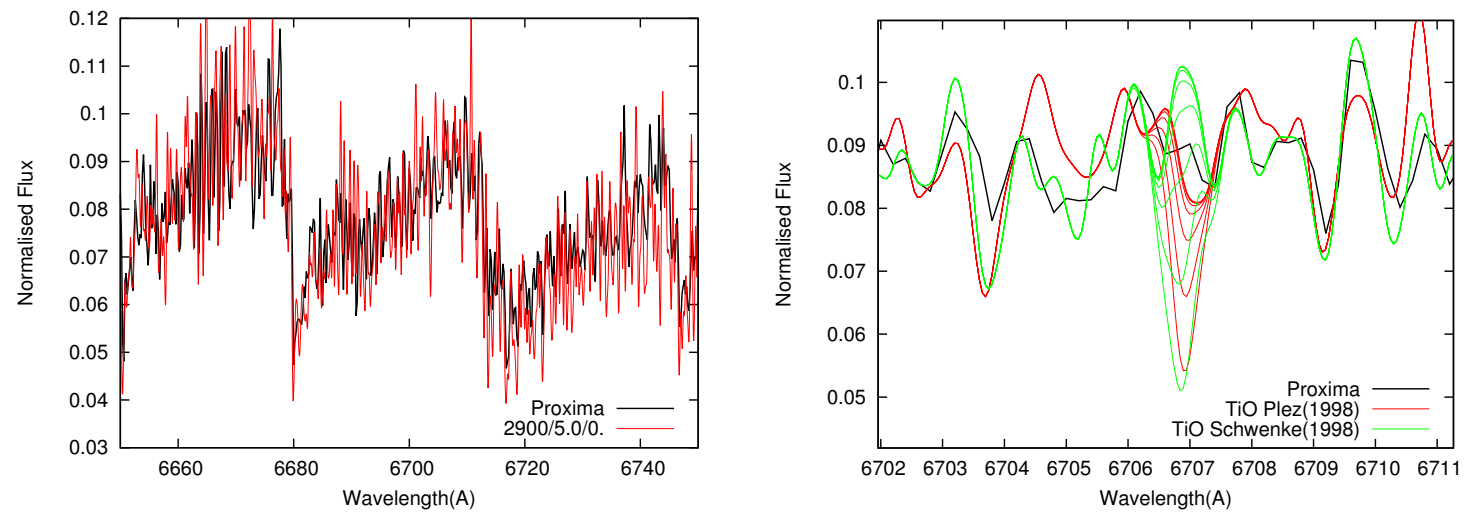

Fig. 4. Left: fit to observed fluxes observed across $6708 \AA$ A. Right: fits of synthetic spectra computed from the line lists of Schwenke (1998) and Plez (1998) for different lithium abundances to the observed $6708 \AA$ blend; lithium abundances from the bottom: $-11.04,-11.54,-12.04 \ldots$ Model atmosphere 2900/5.0/0.

Table 2. Identification of lines in the HARPS spectrum of Proxima shown in the left panel of Fig. 5.

\begin{tabular}{ccccl}
\hline \hline$\lambda$ & Element & $g f$ & $E^{\prime \prime}(\mathrm{eV})$ & Visibility \\
\hline 4147.67 & FeI & $7.87 \mathrm{E}-03$ & 1.485 & Yes \\
4149.76 & FeI & $4.93 \mathrm{E}-06$ & 0.052 & Yes \\
4151.11 & Er I & $2.73 \mathrm{E}+00$ & 0.000 & Yes \\
4152.17 & FeI & $5.86 \mathrm{E}-04$ & 0.958 & Yes \\
4153.90 & FeI & $4.77 \mathrm{E}-01$ & 3.397 & No \\
4154.50 & FeI & $2.05 \mathrm{E}-01$ & 2.832 & No \\
4154.81 & FeI & $3.98 \mathrm{E}-01$ & 3.368 & No \\
4156.80 & FeI & $1.55 \mathrm{E}-01$ & 2.832 & No \\
4159.68 & V I & $1.86 \mathrm{E}-02$ & 0.287 & Yes \\
\hline
\end{tabular}

or absent at these wavelengths, implying that we can see deeper layers of the photosphere of Proxima. However, a comparison of the intensities of observed absorption lines with the computations reveals some problems here:

- A simple analysis of the lines listed in Table 2 shows that only lines with low excitation energy $\left(E^{\prime \prime}<2 \mathrm{eV}\right)$ are seen in the observed photospheric spectrum of Proxima (Fig. 5). We see that the lines with higher excitation energies are weaker (or even absent) in the observed spectrum.

- The strongest atomic lines in the observed spectrum are much stronger in the synthetic spectra computed in the framework of the classical approach. In other words,

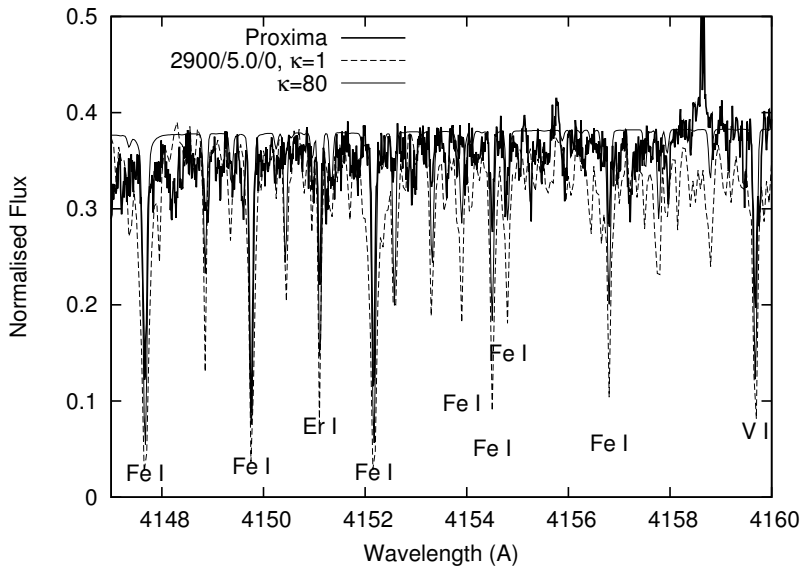

Fig. 5. Identification of absorption lines in the arbitrarily selected part in the observed HARPS spectrum of Proxima.

damping pressure effects are more pronounced in the computed spectrum where atomic lines have more extended wings.

- Our numerical experiments show that changes in effective temperature of $\pm 200 \mathrm{~K}$ or in $\log (\mathrm{g})$ of -0.5 to -1.0 do not improve the fit. We cannot reduce the intensities of saturated lines by reducing the associated abundances because we know that the metallicity of Proxima is near solar (Sect. 3.1.4). 

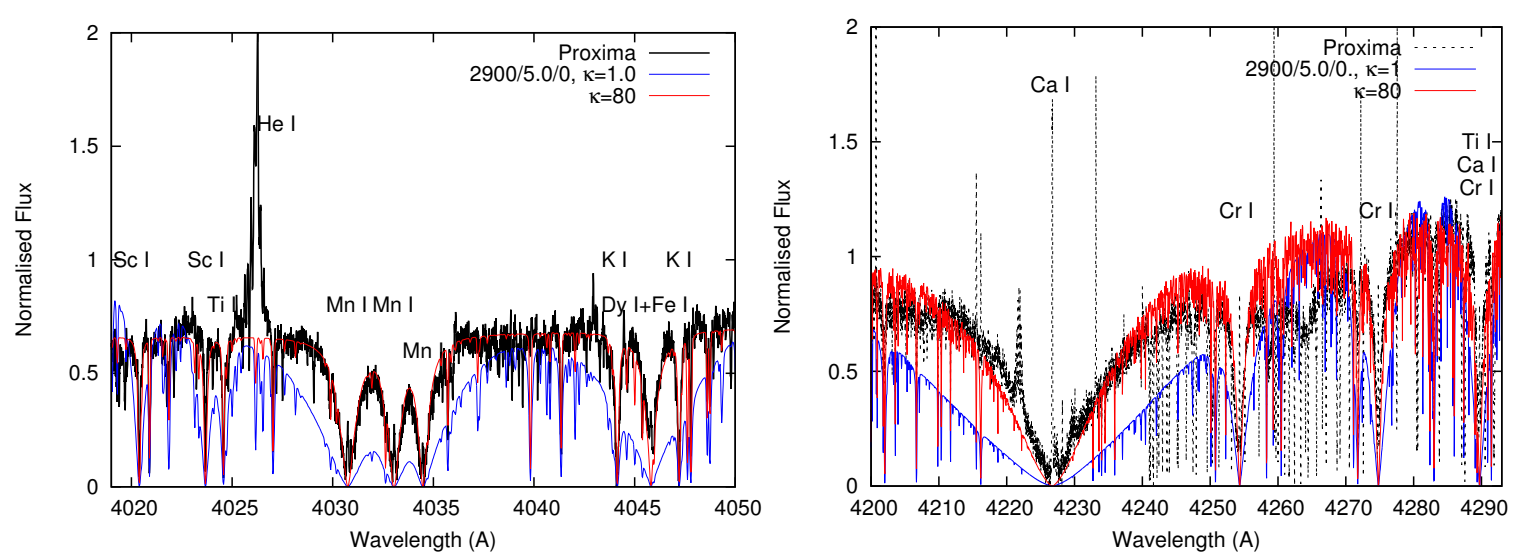

Fig. 6. Spectrum of Proxima computed for the model with enhanced continuum opacity across the blue spectral region.
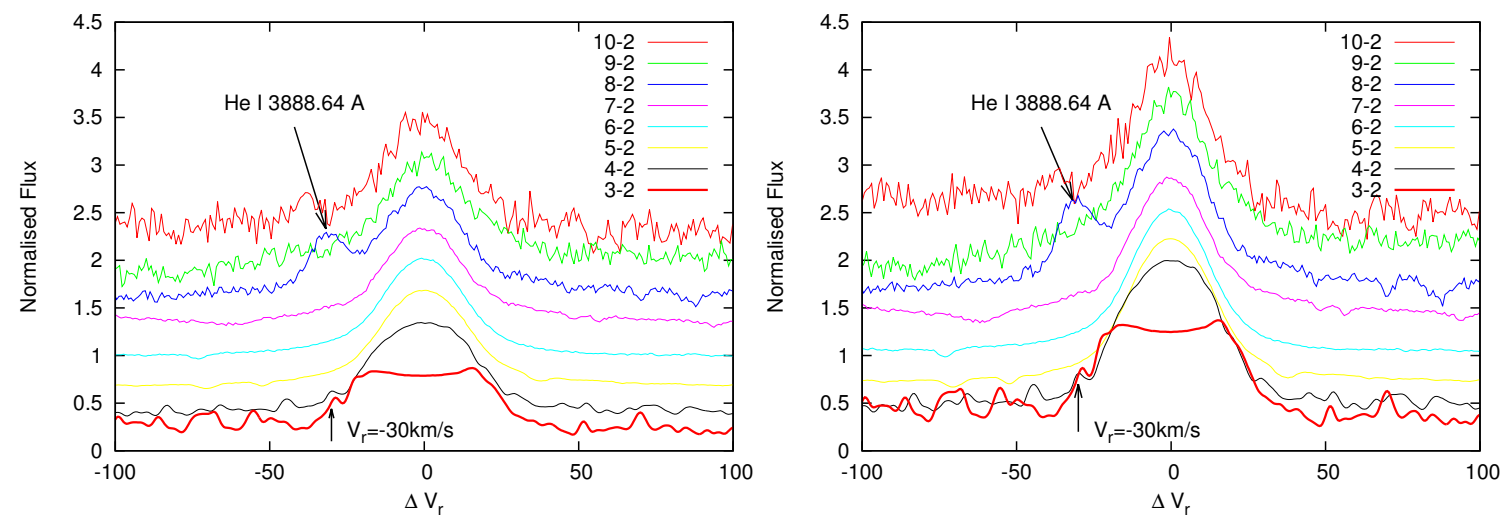

Fig. 7. Profiles of Balmer lines shown in the flux normalised to 1.0 in the centre of lines vs. radial velocity obtained in quiet mode $(l e f t)$ and flare mode (right). To simplify, the plot profiles are shifted in vertical scale and $\mathrm{H}_{\alpha}$ is shown by solid line. The arrows mark the positions of the $V_{r}=$ $-30 \mathrm{~km} \mathrm{~s}^{-1}$ component feature and the He I $3888.64 \AA$ line.

We can explain the differences by enhancing the continuum opacity, as shown in Fig. 6 where we compare the observed spectrum with the newly computed one. To reduce the strength of resonance lines moved the line forming region into lower pressure regions of the atmosphere. In this paper, we use a simple approach suggesting $\chi_{v}^{c}=\chi_{v}^{c 0} \times \kappa$, where $\chi_{v}^{c 0}$ and $\kappa$ are the conventional opacity and adjusting parameter, respectively. Enhancing the continuum opacity across blue spectral range shifts the line forming regions upwards, i.e. to layers of lower pressure. As a consequence, the strongest lines in the computed spectrum show weaker wings, in better agreement with observations. We obtain satisfactory fits after implementing additional continuum opacity in the blue. We can explain the lack of lines of higher excitation potentials as the case where the photosphere of the star moves upwards into layers of the atmosphere with lower temperature where only lines of low excitation potential can form. Absorption lines of lower excitation energies form above the new photosphere, making them less sensitive to these changes (Fig. 5).

\subsection{Emission lines}

The intensity of the emission features visible in the spectra changes during the different activity states of the star. To analyse the difference between the typical activity level and the more quiet states, we created two average spectra representing the typical active state (S) and the quiet state (QC), see Sect. 2.2. The QC spectrum shows lower intensity for most of the measured emission lines, specifically the intensity of $\mathrm{H} \alpha$ here does not exceed $75 \%$ of the maximum emission achieved during strong flares.

\subsubsection{Hydrogen Balmer lines}

All Balmer lines in the spectrum of Proxima are observed in emission. They show strong variability in time with variations up to a factor of 10 in intensity. As a complementary information, we provide a online movie (Movie 1) with the time variability of the $\mathrm{H}_{\alpha}$ in the series of data obtained with HARPS. We measured the pseudo-equivalent widths ( $\mathrm{pEW}$ ) of the emission lines of the full Balmer series in the VLT/X-shooter spectrum with the task splot under IRAF, which we show in Table 3. Interestingly, measurements of pEW of Balmer lines in the averaged HARPS $\mathrm{S}$ spectrum provides values of the same order as those given in the table.

In Fig. 7 we show the intensity profiles of the $\mathrm{H} \alpha$ line vs. Doppler velocity $\left(V_{r}=\Delta \lambda / \lambda \times c\right)$, where $c$ is the speed of light. We show the profiles for both the quiet $(\mathrm{QC}$, left) and flare $(\mathrm{S}$, right) states. In our spectra we see the full Balmer series, from the $\mathrm{H}_{\alpha}$ line to $\mathrm{H}_{\theta}$, corresponding to the 3-2 and 10-2 transitions of the hydrogen atom, respectively. Comparison of their emission profiles provides a few important results:

- All Balmer lines show strong variability responding to the temporal changes of flare activity.

- Self-absorption in the core of the $\mathrm{H}_{\alpha}$ line is observed for almost all stages of activity of Proxima (see online Movie 1). We interpret this phenomenon as evidence for the existence 
Table 3. Central wavelengths $\left(\lambda_{\mathrm{c}}\right)$ and pseudo-equivalent widths measured on the VLT/X-shooter spectrum and expressed in $\AA$ for the observed Balmer series.

\begin{tabular}{cccc}
\hline \hline Line & $j-i$ & $\lambda_{\mathrm{c}}$ & $p E W$ \\
\hline & & $\AA$ & $\AA$ \\
\hline $\mathrm{H} \alpha$ & $3-2$ & $6562.797 \pm 0.02$ & $2.5 \pm 0.5$ \\
$\mathrm{H} \beta$ & $4-2$ & $4861.323 \pm 0.02$ & $5.1 \pm 0.2$ \\
$\mathrm{H} \gamma$ & $5-2$ & $4340.462 \pm 0.02$ & $6.5 \pm 0.5$ \\
$\mathrm{H} \delta$ & $6-2$ & $4101.734 \pm 0.02$ & $9.9 \pm 0.2$ \\
$\mathrm{H} \epsilon$ & $7-2$ & $3970.072 \pm 0.02$ & $5.4 \pm 1.0$ \\
$\mathrm{H} \zeta$ & $8-2$ & $3889.048 \pm 0.02$ & $9.4 \pm 0.8$ \\
$\mathrm{H} \eta$ & $9-2$ & $3835.384 \pm 0.02$ & $3.9 \pm 0.1$ \\
$\mathrm{H} \theta$ & $10-2$ & $3797.898 \pm 0.02$ & $4.7 \pm 0.4$ \\
\hline
\end{tabular}

of comparatively cool matter outside the flare region. The peak of the line on the red side is higher than on the blue, most likely due to the outward motion of the neutral hydrogen as discussed in Fuhrmeister et al. (2011).

- The positions of lines do not change during flare events. We interpret this fact as evidence for a quasi-stationary state of the region where Balmer emission lines form.

- The profiles of all Balmer lines in emission in the intensity vs. doppler velocity parameter space have the same FHWM despite the differences in intensity. The similarity in the profiles of emission lines displayed in Fig. 7 is evidence that they form in the same region of the atmosphere of Proxima heated by flares.

- Some lines in Fig. 7 show a well-pronounced peak at $V_{r}=$ $-30 \mathrm{~km} \mathrm{~s}^{-1}$, which we interpret as an indication of a hot stellar wind moving outwards from the star. This peak is clearly detected in $\mathrm{H}_{\alpha}$ and $\mathrm{H}_{\beta}$, and seen as a wide feature in the blue wings of $\mathrm{H}_{\gamma}-\mathrm{H}_{\epsilon}$.

- In the $\mathrm{H}_{\zeta}$ profile, we again see a well-pronounced emission feature at $V_{r}=-30 \mathrm{~km} \mathrm{~s}^{-1}$; however, in this case we identify it as the He I line at $3889.64 \AA$ (Sect. 4).

- The component blueward shifted does not change much with the activity phase, suggesting that it probably forms in the hot ionised plasma flow (stellar wind) far enough from the flare region of the star.

- When the flare activity rises, the increased emission in $\mathrm{H}_{\alpha}$ covers the line in the blue wing (see online Movie 1). This detail is also seen in Fig. 14 of Fuhrmeister et al. (2011) at lower level activity times.

Photospheric spectra and fluxes show rather marginal responses on the flare activity. In the following we use flux ratios measured in VLT/X-shooter spectra in combination with measurements of pseudo-equivalent widths of $\mathrm{H}_{\alpha}$ and emission features in its blue wing in the HARPS $\mathrm{S}$ and QC spectra, as shown in Fig. 8.

To determine the $\mathrm{H}_{\alpha}$ flux of Proxima received at Earth we followed the procedure by Herbig (1985)

$f_{\mathrm{H}_{\alpha}}=p E W_{\mathrm{H}_{\alpha}} \frac{F_{\lambda}(6563)}{F_{\lambda}(5556)} F_{\lambda}(6563,0.0) 10^{-0.4 V}$,

where the second term is the average flux ratio at the indicated wavelengths for the star. The third term is the VLT/X-shooter flux at $6563 \AA$, received from a star of magnitude $V=0 \mathrm{mag}$, assumed to be $3.8 \times 10^{-9} \mathrm{erg} \mathrm{cm}^{-2} \mathrm{~s}^{-1} \AA^{-1}$. Despite the noise in the $\mathrm{X}$-shooter spectrum at $5556 \AA$, we estimated the second term from the $\mathrm{X}$-shooter spectrum and measured a flux ratio of $\frac{F_{\lambda}(6563)}{F_{\lambda}(5556)}=3 \pm 2$.

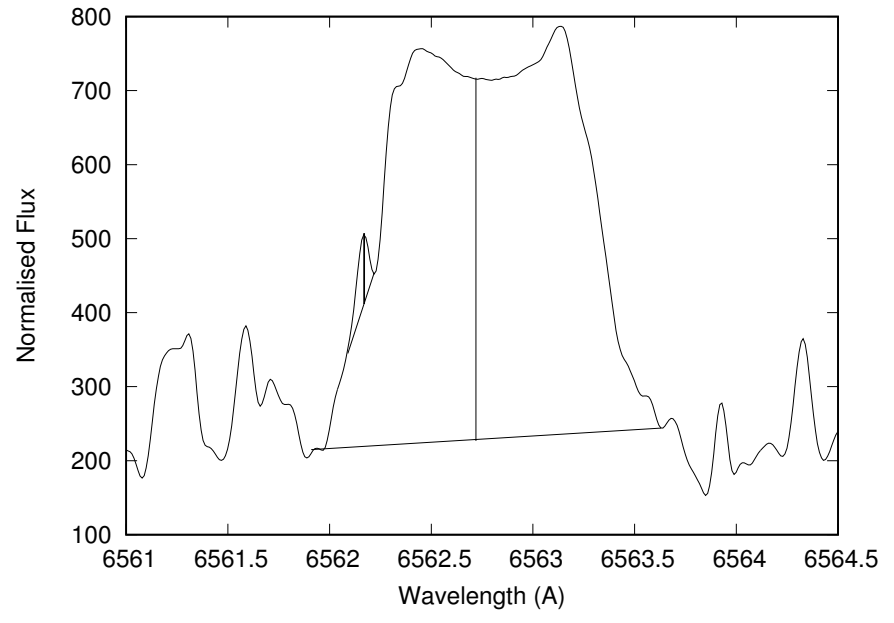

Fig. 8. Scheme of the measurements of the pseudo-equivalent widths of $\mathrm{H}_{\alpha}$ and $V_{r}=-30 \mathrm{~km} \mathrm{~s}^{-1}$ feature in HARPS spectra of Proxima.

The $p E W$ values of $2.7 \pm 0.1$ and $2.6 \pm 0.1 \AA$ were determined in the $\mathrm{S}$ and QC spectra, respectively, with an average value of $p E W_{\mathrm{H}_{\alpha}}=2.65 \pm 0.1 \AA$. For a magnitude of $V=11.13$ for Proxima (Jao et al. 2014), we derived an average value of $f_{\mathrm{H}_{\alpha}}=1.07 \pm 0.710^{-12} \mathrm{erg} \mathrm{cm}^{-2} \mathrm{~s}^{-1}$.

In addition, the second term can be estimated from the $R-I$ colour, using the relationship found by Hodgkin et al. (1995)

$\frac{F_{\lambda}(6563)}{F_{\lambda}(5556)}=2.786(R-I)_{\mathrm{C}}-0.932$,

where $R-I=2.04 \mathrm{mag}$ for Proxima (Jao et al. 2014) and $\frac{F_{\lambda}(6563)}{F_{\lambda}(5556)}=4.75 \pm 0.03$, resulting in $f_{\mathrm{H}_{\alpha}}=1.7 \pm 0.1 \times$ $10^{-12} \mathrm{erg} \mathrm{cm}^{-2} \mathrm{~s}^{-1}$.

As a final result we adopted the mean value of both measurements, resulting in $f_{\mathrm{H}_{\alpha}}=1.4 \pm 0.410^{-12} \mathrm{erg} \mathrm{cm} \mathrm{cm}^{-2} \mathrm{~s}^{-1}$. From this value of $f_{\mathrm{H}_{\alpha}}$, we obtained a luminosity in the $\mathrm{H}_{\alpha}$ of $L_{\mathrm{H}_{\alpha}}=2.8 \pm 0.410^{+26} \mathrm{erg} \mathrm{s}^{-1}$ adopting the distance of $1.30 \mathrm{pc}$ for Proxima from Jao et al. (2014)) and a $L_{\mathrm{H}_{\alpha}} / L_{\mathrm{bol}}=4.5 \pm 0.410^{-5}$ adopting the bolometric luminosity of $6 \times 10^{+30} \mathrm{erg} \mathrm{s}^{-1}$ from Fuhrmeister et al. (2011).

We determined $p E W_{\mathrm{b}}$ of the emission feature seen in the blue emission wing of $\mathrm{H}_{\alpha}$ at $V_{r}=-30 \mathrm{~km} \mathrm{~s}^{-1}$ in the average HARPS spectrum, as shown in Fig. 8. We measured in the $\mathrm{S}$ and QC HARPS spectra $p E W_{\mathrm{b}}=0.018 \AA$, the ratio $p E W_{\mathrm{b}} / p E W_{\mathrm{H}_{\alpha}}=$ 0.007. It allows us to estimate the total energy emitted by the stellar wind in $\mathrm{H}_{\alpha}$

$L_{\mathrm{H}_{\alpha}}^{b}=7.0 \times 10^{-3} \times 2.8 \times 10^{26}=2.0 \times 10^{+24}$.

Assuming complete ionisation of hydrogen in the emitting region we determine the number of emitting $\mathrm{H}_{\alpha}$ atoms, i.e. the lower limit of mass loss,

$\dot{M}=L_{\mathrm{H}_{\alpha}}^{\mathrm{b}} / h v \times m_{\mathrm{p}} \times N_{\mathrm{sec}}=3.7 \times 10^{19} \mathrm{~g} \mathrm{yr}^{-1}=1.8 \times 10^{-14} M_{\odot} \mathrm{yr}^{-1}$,

where $m_{\mathrm{p}}$ and $N_{\mathrm{sec}}$ are the mass of $\mathrm{H} \mathrm{I}$ atom and the number of $\mathrm{sec}$ in $1 \mathrm{yr}$.

\subsubsection{Hel line at 4026.19 and $3888.64 \AA$}

The HeI line at $4026.19 \AA$ was identified by Fuhrmeister et al. (2011, see their Fig. 13 and Table 4). We observe strong variability of this line in the observed spectra. We note that the HeI line 
Table 4. Spectroscopic parameters of the HeI emission lines.

\begin{tabular}{cccc}
\hline \hline $\begin{array}{c}\text { Wavelength } \\
(\mathrm{A})\end{array}$ & Terms & $j^{\prime \prime}-j^{\prime}$ & $\begin{array}{c}E^{\prime \prime}-E^{\prime} \\
\left(\mathrm{cm}^{-1}\right)\end{array}$ \\
\hline 4026.18436 & $3 \mathrm{P}_{0}-3 \mathrm{D}$ & $2-1$ & $169086.87-193917.26$ \\
4026.18590 & $3 \mathrm{P}_{0}-3 \mathrm{D}$ & $2-2$ & $169086.87-193917.26$ \\
4026.18600 & $3 \mathrm{P}_{0}-3 \mathrm{D}$ & $2-3$ & $169086.87-193917.25$ \\
4026.19675 & $3 \mathrm{P}_{0}-3 \mathrm{D}$ & $1-1$ & $169086.95-193917.26$ \\
4026.19829 & $3 \mathrm{P}_{0}-3 \mathrm{D}$ & $1-2$ & $169086.95-193917.26$ \\
4026.35695 & $3 \mathrm{P}_{0}-3 \mathrm{D}$ & $0-1$ & $169087.93-193917.26$ \\
& & & \\
3888.60467 & $3 \mathrm{~S}-3 \mathrm{P}_{0}$ & $1-0$ & $159856.08-185564.96$ \\
3888.64560 & $3 \mathrm{~S}-3 \mathrm{P}_{0}$ & $1-1$ & $159856.08-185564.69$ \\
3888.64893 & $3 \mathrm{~S}-3 \mathrm{P}_{0}$ & $1-2$ & $159856.08-185564.67$ \\
\hline
\end{tabular}

Table 5. Observed components of the HeI 4026 Å emission line.

\begin{tabular}{ccc}
\hline \hline $\mathrm{NN}$ & $\begin{array}{c}\Delta \lambda \\
(\AA)\end{array}$ & $\begin{array}{c}V_{r} \\
\left(\mathrm{~km} \mathrm{~s}^{-1}\right)\end{array}$ \\
\hline 1 & -0.560 & -41.73 \\
2 & -0.420 & -31.29 \\
3 & -0.240 & -17.88 \\
4 & -0.080 & -4.59 \\
5 & 0.090 & 6.71 \\
6 & 0.220 & 16.39 \\
7 & 0.380 & 28.32 \\
\hline
\end{tabular}

has the highest excitation energy $\left(E^{\prime \prime}=20.97 \mathrm{eV}\right.$; see Table 5) with respect to other emission lines observed across our spectral range. It mostly likely formed in the outermost layers of the atmosphere heated by shock waves. Indeed, the HeI line cannot form in the same place where emissions in the cores of absorption lines of neutral metals form. Furthermore, the HeI emission line is broader.

In the QC dataset, the HeI line shows a multiplet structure and looks more intensive in comparison with the $\mathrm{S}$ state (Fig. 9). We suggest that the strong flares destroy the extended emitted region where the line is formed. Likely, broad component seen in the $\mathrm{S}$ spectrum can be associated with a flare region, where, by definition, the dispersion of velocities is larger. In the more quiet modes, we see a few shells moving outwards from the star represented by components equally shifted bluewards and redwards suggesting a multicomponent for the line (see Table 5).

Another $\mathrm{He} \mathrm{I}$ line is seen in the blue wing of the hydrogen $\mathrm{H}_{\zeta}$ line, at $3888.64 \AA$. The excitation potential of this line is only a bit lower $(19.82 \mathrm{eV})$ than the $4026 \AA$ line (see Table 4). Neither line shows any remarkable wavelength shift, so we may assume they form in the same extended quasi-stable hot layers.

\subsection{Emission cores of resonance lines of atoms and ions}

\subsection{1. $\mathrm{H}$ and $\mathrm{K}$ of $\mathrm{Ca}$ II}

The CaII $\mathrm{H}$ and $\mathrm{K}$ lines are well known as indicators of stellar activity. These lines are collisional controlled and respond to an increase in the temperature of the lower chromosphere of quiet stars like the Sun or Arcturus (Ayres \& Linsky 1975). Hence, the appearance of these lines in the spectrum of Proxima is the evidence of chromosphere. Moreover, these lines are extremely strong:

- emission lines of $\mathrm{H}$ and $\mathrm{K} \mathrm{CaII} \mathrm{fill} \mathrm{the} \mathrm{broad} \mathrm{absorption} \mathrm{lines} \mathrm{seen}$ in less active M dwarfs of the same spectral type, such as GJ699 (Barnard star), as shown in Fig. 10;

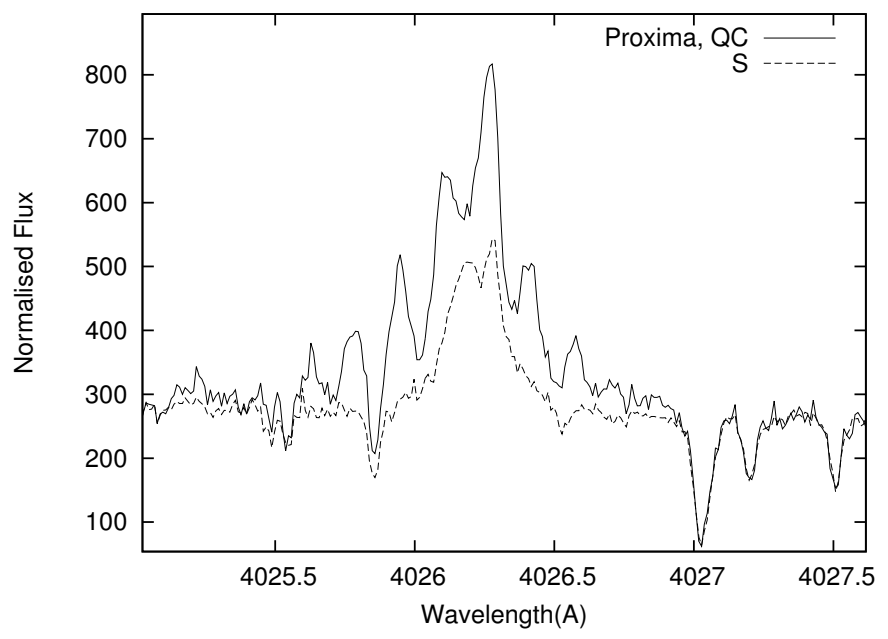

Fig. 9. Profiles of the HeI $4026 \AA$ line observed in the $\mathrm{S}$ and QC states of flare activity.

- these emission lines do not show any wavelength shift with respect to the photospheric lines;

- the emission profiles of the $\mathrm{H}$ and $\mathrm{K}$ lines are more intense in the quiet states, in contrast to what we see for Balmer lines of hydrogen. Big flares likely affect the regions of their formation;

- the self-absorption seen in the cores of the CaII H and $\mathrm{K}$ lines indicates that the temperature drops at the upper boundary of their formation region;

- the intensity of their components changes in time and with activity, as demonstrated in the online Movie 2.

\subsection{2. $\mathrm{Na} \mathrm{H}$ and $\mathrm{K}$ resonance lines}

Strong emission $\mathrm{H}$ and $\mathrm{K}$ resonance lines of sodium are notable features in the observed spectra of Proxima. These lines are controlled by photoelectric processes (Thomas 1959). For these lines, the ratio of the photo-ionisation sink to the collisional sink is much higher than 1 (see Athay 1972). Therefore, these lines, like the hydrogen lines, show a rather marginal response to the temperature gradients present in chromospheres. We refer the reader to the response of the lithium line on chromospheric-like structures in the atmosphere of $\mathrm{M}$ dwarfs described in Pavlenko (1998b). Only extreme cases of chromospheric activity can provide emission cores in the photo-electrically controlled lines, which is most likely the case of Proxima.

In Fig. 11, we compared the profiles of the $\mathrm{K}$ and $\mathrm{H}$ lines of the $\mathrm{NaI}$ resonance doublet, which indicate a few non-trivial results:

- the strong emission cores seen in the NaI resonance doublet show significant changes in total emitted energy (see online Movie 3);

- however, the dispersion of velocities in the line forming region change very little with the activity level. In the more quiet mode (QC), emission profiles of both components are a bit narrower, while flares increase the width of the emitted lines. Likely, the resonance doublet of $\mathrm{NaI}$ forms in the chromosphere of the star.

- the profiles of strong photospheric absorption lines show almost no response on the level of activity, suggesting that photospheric layers are not bound or are weakly bound with regions governed by stellar activity processes.

\subsubsection{Other emission lines}

In Table A.1 we provide the list of other emission lines with emission cores seen in the spectrum of Proxima. We find emission lines of many different elements, from HeI $(z=2)$ to Dy $(z=66)$. Our list is more complete than the one given in Fuhrmeister et al. (2011) because of the higher resolution of our spectra. Moreover, our list contains both pure chromospheric lines and lines of very high excitation like HeI, which 

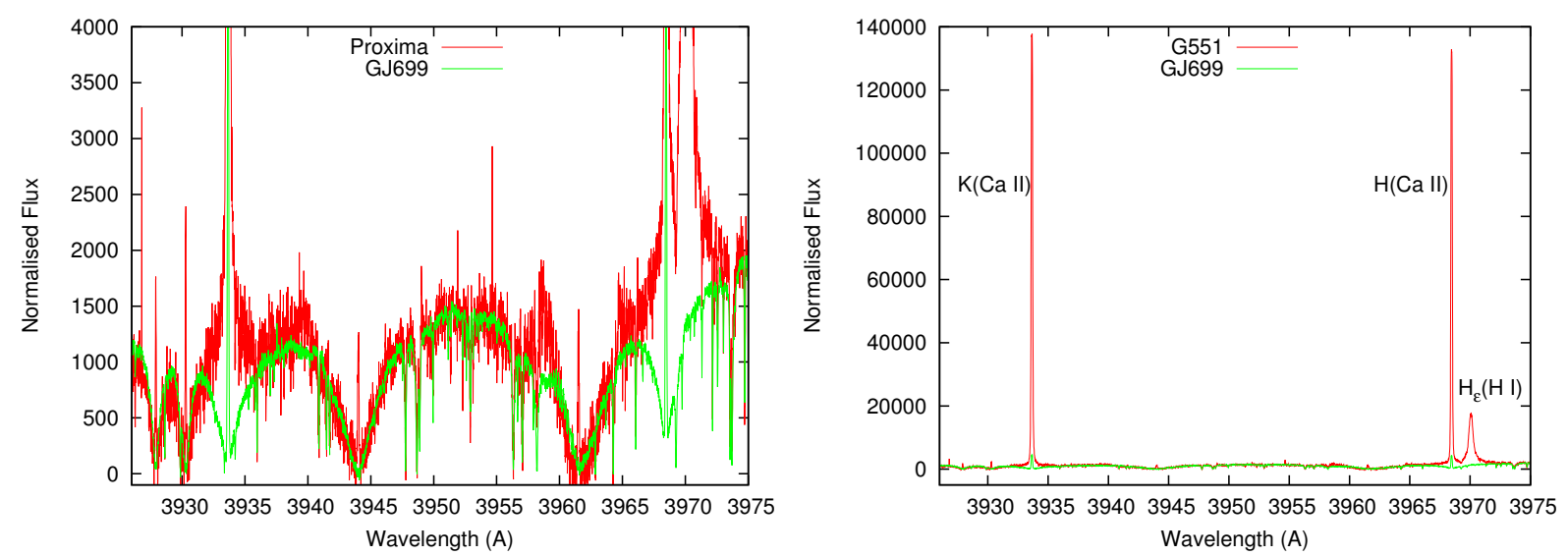

Fig. 10. Left: emission lines in the spectral region of the Ca II H and K lines of the star Proxima and GJ 699 (see Sect. 3.3.2). Right: same plot shown over a wider wavelength range. The GJ699 spectrum shown here was downloaded from the HARPS archive.
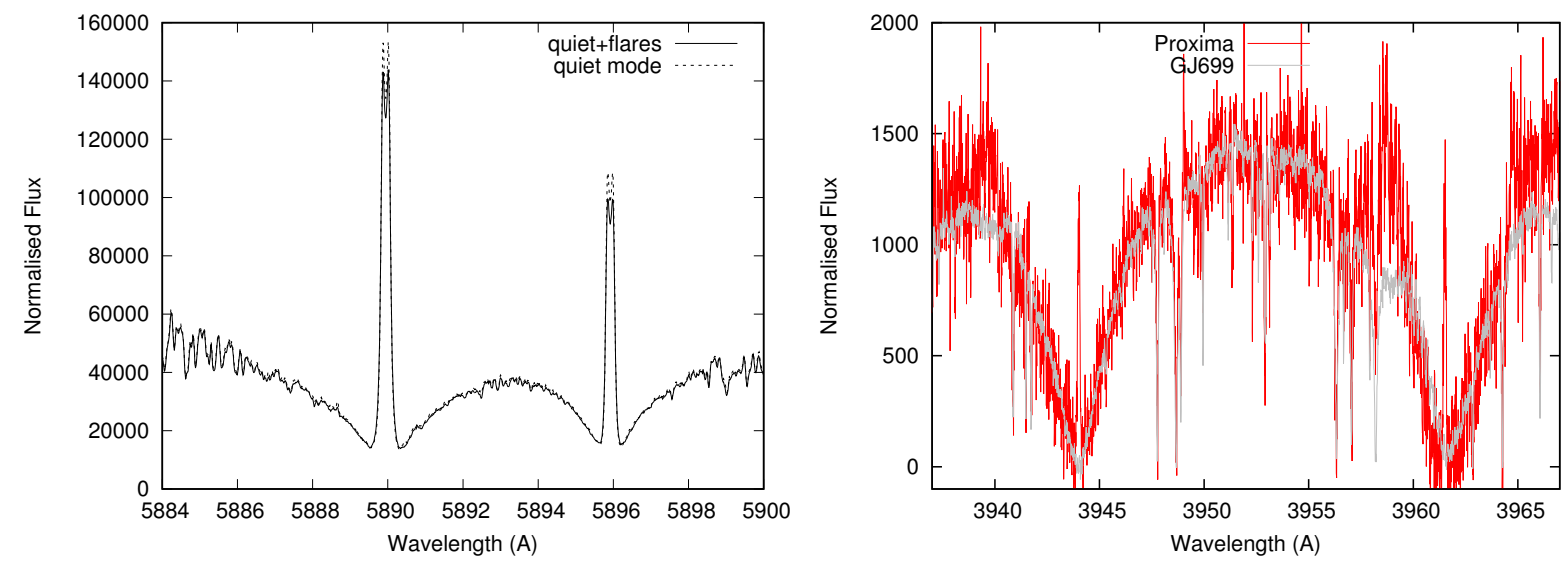

Fig. 11. Left: resonance doublet of $\mathrm{NaI}$ at different levels of activity. Right: resonance doublet of AlI in comparison with GJ 699, both shown in quiet state.
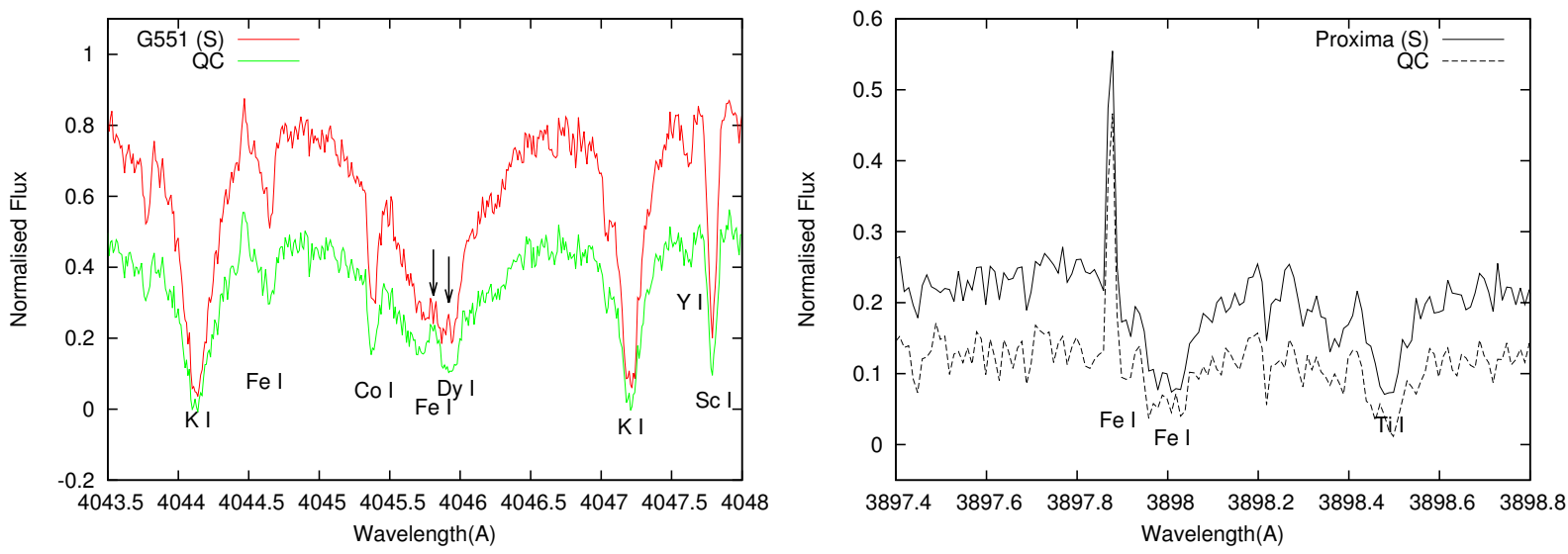

Fig. 12. Left: absorption lines of the neutral metals with emission cores. The left arrow at $4045.81 \AA$ marks the self-absorption in the core of the strong Fe I absorption line. In the S spectrum the strong emission detail in the core of the nearby Dy I $\lambda 4045.97 \AA$ line is marked by the right arrow. In the QC spectrum the detail is "eaten" by self-absorption. Self-absorption in the weak emission core of K I line at $\lambda 4047.21$ is seen as well. Right: example of iP Cyg profiles.

can be formed in areas governed by shock waves (i.e. formed outside the chromosphere). We can distinguish them by the widths of their observed profiles because chromospheric lines formed in the cores of absorption lines are narrower than the lines of hydrogen and helium formed in the layers with larger velocity dispersion.

We assign specific labels to some of the lines as follows:

- ec-absorption lines with emission cores;
- ecs - emission cores with self-absorption as shown in the left panel of Fig. 12;

- P Cyg and iP Cyg - absorption lines with emission components in the red and blue wings, respectively. An inverse P Cyg line is shown in the right panel of Fig. 12.

We generally find that most of the strong resonance lines show emission cores and are often shifted with respect to the central wavelengths. 
Observed emission cores do not form in the spherically symmetrical and stable atmosphere. Moreover, the strong temporal changes of the emission lines provide evidence that the layers of the chromosphere where they form are strongly affected by the flaring processes. Some other cases show P Cyg or inverse P Cyg profiles. These phenomena likely reflect the complicated dynamical processes occurring in the atmosphere of Proxima.

\section{Discussion on the atmosphere of Proxima}

\subsection{Photosphere}

The analysis of the VLT/X-shooter spectra obtained with intermediate resolution shows that the atmosphere of Proxima in the optical and NIR spectral range is similar to the rest of normal $\mathrm{M}$ dwarfs of the same spectral classes M5-M6. The optical spectral range is governed by the Ti and VO bands; the NIR is dominated by molecular bands of water. Our theoretical spectra computed for a model atmosphere of $T_{\text {eff }}=2900 \mathrm{~K}$ fits the observed spectral energy distribution well enough in agreement with the results of Rajpurohit et al. (2013) and Passegger et al. (2016) In summary, we obtain rather good fits of our synthetic spectra computed for the canonical PHOENIX model atmosphere of solar metallicity across all spectral ranges observed by the VLT/X-shooter, except for slightly lower fluxes in the spectral region of the $K$ band, which seems to be a common problem of similar mid-M dwarf investigations (see Pavlenko et al. 2006a).

Our computed spectral energy distributions depend on log g rather marginally; still we note a weak trend toward lower log $\mathrm{g}$ in accordance with $\log g=4.5$ found by Mann et al. (2015). On the other hand, strong alkali lines provide a clear response on log g changes. We obtained better fits to profiles of some observed absorption lines of $\mathrm{Na}$, $\mathrm{Rb}$, and the subordinate triplet of $\mathrm{Na}$ at $8200 \AA$ with $\log g=5.0$ (see also Rajpurohit et al. 2013), instead of $\log g=5.5$, as obtained by Passegger et al. (2016). Our result is in agreement with the $\log g=$ $5.23 \pm 0.14$ derived from the interferometric measurement of the Proxima radius and mass-radius relations by Demory et al. (2009).

The opacity of TiO bands around $4200 \AA$ starts to decrease bluewards (Pavlenko 2014). At shorter wavelengths, we explore deeper regions of the atmosphere of Proxima, where the observed spectrum is dominated by absorption lines of neutral atoms. The resonance lines of neutral species become very strong. Theoretical spectra provide wings that are too strong, due to the effects of pressure damping which broadens these lines. In the case of Proxima we obtain the best solution for $\kappa=80$. This value looks very high, but we work in the regime of low temperatures where the conventional opacity is extremely low in this case, due to the low density of the $\mathrm{H}^{-}$ion, which is the main source of opacity in the atmospheres of M stars. In other words, we should find larger electron densities to increase opacity in the low photospheric layers. We suggest that this effect may be created by the overionisation of alkali metals, which are the main donors of free electrons. Indeed, photons with $\lambda<4200 \AA$ can ionise the neutral atoms in latetype atmosphere, due to the low electron densities where processes of recombination are not so effective. The additional opacity affects only the emitted spectrum in the UV and blue wavelengths, occurring deep in the atmosphere. The temperature structure of the upper layers of the model atmosphere are mainly determined by molecular opacities, which explains why we can reliably fit the spectral energy distribution of Proxima at longer wavelengths.

On the other hand, for some spectral diagnostics, it is also possible that the analysis is too simplified and 1D LTE syntheses may indeed underestimate some of the opacity (lines) in the blue. More complex analysis involving 3D atmospheres from hydro simulations sometimes find that the missing opacity is even greater in 3D NLTE models than in 1D LTE. There are hints in the paper by Fuhrmeister et al. (2011) that much more sophisticated 3D atmospheres should be applied in the case of Proxima.

\subsection{Chromosphere}

The chromosphere represents the extended part of the atmosphere of Proxima, where narrow emission lines of different intensities vary with time. We observe temporal variations in these lines because of interactions between the flares and the chromosphere. We know that solar flares originate in high chromosphere-corona regions. The strongest flares move downwards and go deeper into the photosphere. However, our movies show mainly temporal changes of intensity of chromospheric lines, while the photospheric spectrum shows marginal response to flares. In most cases, even the strongest flares seem to occur in local regions far above the surface/photosphere of Proxima. The TiO line forming region is separated from the hot flare regions by the mantle of the cool plasma. Nevertheless, the chromosphere of Proxima is very powerful because it shows many absorption lines of neutral metals with narrow emission cores. These emission cores most likely form in the chromosphere above the temperature minimum. The presence of emission cores within the lines of neutral alkalies and neutral atoms which are fully controlled by radiation can only occur in the presence of steep temperature gradients in the chromosphere (see e.g. NLTE simulations of emission cores of Li in Pavlenko 1998a).

\subsection{Flares}

Hydrogen Balmer lines in emission is a common phenomenon in the Sun. Balmer lines are controlled by photo-electrical processes and are not sensitive to the temperature structure of the atmosphere. Their emission is mainly the result of ionisation and collisional processes created by shock waves following a flare event. The hydrogen emission lines seen in the Proxima spectrum form mainly in flare regions. They are broader than chromospheric lines of CaII, NaI, and other neutral metals. This effect most likely results from a larger scatter in their velocity distribution, as seen in Fig. 10, where we compare the CaII and $\mathrm{H}_{\epsilon}$ lines: the CaII line is much stronger, but $\mathrm{H}_{\epsilon}$ is broader.

\subsection{Stellar wind}

We observe a quasi-stationary component in the blue wing of the $\mathrm{H}_{\alpha}$ and $\mathrm{H}_{\beta}$ emission in the HARPS spectra. We interpret its presence as a result of the flow of highly ionised plasma with a velocity of $V_{r}=-30 \mathrm{~km} \mathrm{~s}^{-1}$. We assume that the observed component most likely relates to the hot stellar wind outflow generated by the high level of flare activity in Proxima. From our estimate of the total energy emitted in the $\mathrm{H}_{\alpha}$ line, we infer a lower limit of the mass loss of $\dot{M}=1.8 \times 10^{-14} M_{\odot} \mathrm{yr}^{-1}$ because we do not consider emitted energies by other emission lines.

Wood et al. $(2000,2001)$ estimated a four or six times lower mass loss of $\dot{M} \leq 0.2 \dot{M}_{\odot}=0.510^{-14} M_{\odot} \mathrm{yr}^{-1}$ or $0.310^{-14} M_{\odot} \mathrm{yr}^{-1}$ for the cases of average solar wind mass loss $\dot{M}_{\odot}=1.374 \times 10^{12} \mathrm{~g} / \mathrm{s}=$ $2.3 \times 10^{-14} M_{\odot} \mathrm{yr}^{-1}$ (Hundhausen 1997) and $\dot{M}_{\odot} \sim 1.3 \times 10^{-14} M_{\odot} \mathrm{yr}^{-1}$ (Goldstein et al. 1996), respectively. Nevertheless, measurements by Wood et al. $(2000,2001)$ are based on the analysis of the $L_{\alpha}$ absorption, which relates to absorption by neutral hydrogen atoms. Both estimates relate to different parts of the stellar wind. It is worth noting that the level of activity and, respectively, mass loss for the late-type stars should change in time. Indeed, we observe different levels of activity in the M dwarf population of our Galaxy. The spectrum of GJ699 used in our paper for the comparison with Proxima provides clear evidence of much lower levels of activity. Likely, Proxima is currently passing its evolutionary epoch of high activity.

In our spectra we see manifestations of cool and hot components of the stellar wind from Proxima. Cool neutral hydrogen located above the flare region provides the asymmetrical self-absorption in $\mathrm{H}_{\alpha}$ core, as discussed in Fuhrmeister et al. (2011). We defer a more detailed analysis of this phenomenon to a future paper.

\section{Conclusions}

In the framework of our work a few results were obtained:

- From the fits of spectral energy distributions observed in the optical and near-IR spectral ranges, we obtained effective temperature of Proxima: $\quad T_{\text {eff }}=2900 \pm 100 \mathrm{~K}$. 
- Fits to profiles of strong atomic lines observed in the optical spectrum of Proxima provide good restrictions for the gravity in atmosphere $\log g=5.0 \pm 0.25$.

- From the analysis of strong resonance and subordinate lines of $\mathrm{Na}$, $\mathrm{K}, \mathrm{Rb}$, and from lines of intermediate strengths of $\mathrm{Ti} \mathrm{I}$ and $\mathrm{Fe} \mathrm{I}$ lines formed at the background of $\mathrm{TiO}$ and $\mathrm{VO}$ bands, we obtained solar abundances of these elements in the atmosphere of Proxima.

- From the fits to the observed spectrum across the Li resonance doublet, we determined the upper limit of $\mathrm{Li}$ abundance $\log N(\mathrm{Li})=$ -12.04 , which is consistent with the expected depletion of an old fully convective low-mass star.

- Photospheric lines observed in the optical and infrared spectra of Proxima can be fitted by standard synthetic models. However, observed strong lines of low excitation potentials in the blue spectral region show narrower profiles than expected indicating a formation in lower pressure layers in the atmosphere. We were able to reproduce their profiles by incorporating additional opacity, which shifts their formation layers upwards, in the lower pressure regions.

- In spite of a comparatively high level of activity, we found that the photospheric spectrum shows rather marginal response on the flare activity of Proxima, except for very strong flares.

- The emission lines of hydrogen are good indicators of stellar activity. At the times of strong flares they become more intense. On the contrary, strong emission lines formed in the chromosphere, i.e. $\mathrm{H} \& \mathrm{~K} \mathrm{Ca}$ II, H\&K Na I, reduce their intensity at the occurrence of strong flares. Likely, strong flares change the structure of chromosphere. These chromospheric originated lines are narrower than the hydrogen lines formed in the flare region.

- The He I line at $4026.19 \AA$ is observed in emission and also reduces its intensity in the presence of strong flares. Due to the larger excitation potential of the He I line, it should form in hotter layers above the hydrogen line formation region. In the absence of strong flares, the He I emission line at $4026.19 \AA$ shows a multicomponent profile. Likely, it reflects complicate structure of the line forming region. In the S spectrum the intensity of the He I lines is lower than in QC, which means that the He I lines formation layers are affected by flares.

- In the blue wing of the hydrogen $\mathrm{H}_{\alpha}$ and $\mathrm{H}_{\beta}$ emission lines we found an emission component shifted to $V_{r}=30 \mathrm{~km} \mathrm{~s}^{-1}$ with respect to their cores. We interpret the emission components as evidence of the hot stellar wind from Proxima. Using the simple model of complete ionisation of hydrogen atoms in the stellar wind we estimate a minimum mass loss of $\dot{M}=1.8 \times 10^{-14} M_{\odot} \mathrm{yr}^{-1}$.

Acknowledgements. This research is based on observations collected at the European Organisation for Astronomical Research in the Southern Hemisphere under ESO programme(s) 087.D-0300(A). This research has made use of the services of the ESO Science Archive Facility. Y.P. is grateful for financial support from the Fundación Jesús Serra for a two-month stay (Sept.-Oct. 2016) as a visiting professor at the Instituto de Astrofísica de Canarias (IAC) in Tenerife. N.L. and V.J.S.B. are supported by the AYA2015-69350-C3-2-P program from the Spanish Ministry of Economy and Competitiveness (MINECO). A.S.M. acknowledges financial support from the Swiss National Science Foundation (SNSF) J.I.G.H. acknowledges financial support from the Spanish MINECO under the 2013 Ramón y Cajal program MINECO RYC-2013-14875, and A.S.M., J.I.G.H., and R.R.L. also acknowledge financial support from the Spanish ministry project MINECO AYA2014-56359-P. The authors kindly thank M.A. Bautista, S.N Nahar, M.J. Seaton, and D.A. Verner who supplied data compiled in the NIST database. This research has made use of the Simbad and Vizier databases, operated at the Centre de Données Astronomiques de Strasbourg (CDS), of the VALD database, operated at Uppsala University, the Institute of Astronomy RAS in Moscow, the University of Vienna, and of NASA's Astrophysics Data System Bibliographic Services (ADS). We thank the anonymous referee for the thorough review, and we greatly appreciate the comments and suggestions which significantly contributed to improving the quality of the publication.

\section{References}

Anders, E., \& Grevesse, N. 1989, Geochim. Cosmochim. Acta, 53, 197 Anglada-Escudé, G., Amado, P. J., Barnes, J., et al. 2016, Nature, 536, 437 Asplund, M., Grevesse, N., Sauval, A. J., \& Scott, P. 2009, ARA\&A, 47, 481 Athay, R. G. 1972, Geophysics and Astrophysics Monographs, Vol. 1 (Dordrecht: D. Reidel)
Ayres, T. R., \& Linsky, J. L. 1975, ApJ, 201, 212

Benedict, G. F., McArthur, B., Nelan, E., et al. 1998, AJ, 116, 429 Bessell, M. S. 1991, AJ, 101, 662

Bonfils, X., Gillon, M., Forveille, T., et al. 2011, A\&A, 528, A111

Charbonneau, D., Berta, Z. K., Irwin, J., et al. 2009, Nature, 462, 891

Christian, D. J., Mathioudakis, M., Bloomfield, D. S., Dupuis, J., \& Keenan, F. P. 2004, ApJ, 612, 1140

Davenport, J. R. A., Kipping, D. M., Sasselov, D., Matthews, J. M., \& Cameron, C. 2016, ApJ, 829, L31

Demory, B.-O., Ségransan, D., Forveille, T., et al. 2009, A\&A, 505, 205

D’Odorico, S., Dekker, H., Mazzoleni, R., et al. 2006, in SPIE Conf. Ser., 6269

Fuhrmeister, B., Lalitha, S., Poppenhaeger, K., et al. 2011, A\&A, 534, A133

Garraffo, C., Drake, J. J., \& Cohen, O. 2016, ApJ, 833, L4

Goldstein, B. E., Neugebauer, M., Phillips, J. L., et al. 1996, A\&A, 316, 296

Gomes da Silva, J., Santos, N. C., Bonfils, X., et al. 2011, A\&A, 534, A30

Grevesse, N., \& Sauval, A. J. 1998, Space Sci. Rev., 85, 161

Herbig, G. H. 1985, ApJ, 289, 269

Hodgkin, S. T., Jameson, R. F., \& Steele, I. A. 1995, MNRAS, 274, 869

Hundhausen, A. J. 1997, in Cosmic Winds and the Heliosphere, eds. J. R. Jokipii,

C. P. Sonett, \& M. S. Giampapa (Tucson: University of Arizona Press), 259

Ivanyuk, O. M., Jenkins, J. S., Pavlenko, Y. V., Jones, H. R. A., \& Pinfield, D. J. 2017, MNRAS, 468, 4151

Jao, W.-C., Henry, T. J., Subasavage, J. P., et al. 2014, AJ, 147, 21

Kervella, P., Thévenin, F., Ségransan, D., et al. 2003, A\&A, 404, 1087

Kervella, P., Thévenin, F., \& Lovis, C. 2017, A\&A, 598, L7

Kirkpatrick, J. D., Gelino, C. R., Cushing, M. C., et al. 2012, ApJ, 753, 156

Kurucz, R. L., Furenlid, I., Brault, J., \& Testerman, L. 1984, Solar flux atlas from 296 to $1300 \mathrm{~nm}$ (New Mexico: National Solar Observatory)

Lurie, J. C., Henry, T. J., Jao, W.-C., et al. 2014, AJ, 148, 91

Mann, A. W., Feiden, G. A., Gaidos, E., Boyajian, T., \& von Braun, K. 2015, ApJ, 804, 64

Mayor, M., Pepe, F., Queloz, D., et al. 2003, The Messenger, 114, 20

Noyes, R. W., Hartmann, L. W., Baliunas, S. L., Duncan, D. K., \& Vaughan, A. H. 1984, ApJ, 279, 763

Passegger, V. M., Wende-von Berg, S., \& Reiners, A. 2016, A\&A, 587, A19

Pavlenko, Y. V. 1998a, Astron. Rep., 42, 501

Pavlenko, Y. V. 1998b, Astron. Rep., 42, 787

Pavlenko, Y. V. 2003, Astron. Rep., 47, 59

Pavlenko, Y. V. 2014, Astron. Rep., 58, 825

Pavlenko, Y. V., \& Jones, H. R. A. 2002, A\&A, 396, 967

Pavlenko, Y. V., \& Schmidt, M. 2015, Kinemat. Phys. Celest. Bodies, 31, 90

Pavlenko, Y. V., Rebolo, R., Martin, E. L., \& Garcia Lopez, R. J. 1995, A\&A, 303, 807

Pavlenko, Y. V., Jones, H. R. A., Lyubchik, Y., Tennyson, J., \& Pinfield, D. J. 2006a, A\&A, 447, 709

Pavlenko, Y. V., van Loon, J. T., Evans, A., et al. 2006b, A\&A, 460, 245

Plez, B. 1998, A\&A, 337, 495

Quintana, E. V., \& Barclay, T. 2014, in AAS Meeting Abstracts, 224, 113.06

Rajpurohit, A. S., Reylé, C., Allard, F., et al. 2013, A\&A, 556, A15

Reipurth, B., \& Mikkola, S. 2012, Nature, 492, 221

Ribas, I., Bolmont, E., Selsis, F., et al. 2016, A\&A, 596, A111

Rivera, J. L., Loinard, L., Dzib, S. A., et al. 2015, ApJ, 807, 119

Ryabchikova, T., \& Pakhomov, Y. 2015, Baltic Astron., 24, 453

Ryabchikova, T., Piskunov, N., Kurucz, R. L., et al. 2015, Phys. Sci., 90, 054005

Schwenke, D. W. 1998, Faraday Discussions, 109, 321

Suárez Mascareño, A., Rebolo, R., González Hernández, J. I., \& Esposito, M. 2015, MNRAS, 452, 2745

Suárez Mascareño, A., Rebolo, R., González Hernández, J. I., \& Esposito, M. 2016, MNRAS, 457, 2604

Thompson, A. P. G., Watson, C. A., de Mooij, E. J. W., \& Jess, D. B. 2017, MNRAS, 468, L16

Tody, D. 1986, in SPIE Conf. Ser., Vol. 627, ed. D. L. Crawford, 733

Tody, D. 1993, in Astronomical Data Analysis Software and Systems II, eds.

R. J. Hanisch, R. J. V. Brissenden, \& J. Barnes, ASP Conf. Ser., 52, 173

Torres, G., Kipping, D. M., Fressin, F., et al. 2015, ApJ, 800, 99

Turbet, M., Leconte, J., Selsis, F., et al. 2016, A\&A, 596, A112

Udry, S., Bonfils, X., Delfosse, X., et al. 2007, A\&A, 469, L43

Vernet, J., Dekker, H., D'Odorico, S., et al. 2011, A\&A, 536, A105

Wargelin, B. J., Saar, S. H., Pojmański, G., Drake, J. J., \& Kashyap, V. L. 2017, MNRAS, 464, 3281

Wertheimer, J. G., \& Laughlin, G. 2006, AJ, 132, 1995

Wood, B. E., Linsky, J. L., Mueller, H.-R., \& Zank, G. P. 2000, BAAS, 197, 1406

Wood, B. E., Linsky, J. L., Müller, H.-R., \& Zank, G. P. 2001, ApJ, 547, L49

Wright, D. J., Wittenmyer, R. A., Tinney, C. G., Bentley, J. S., \& Zhao, J. 2016, ApJ, 817, L20 


\section{Appendix A: Additional figures and tables}
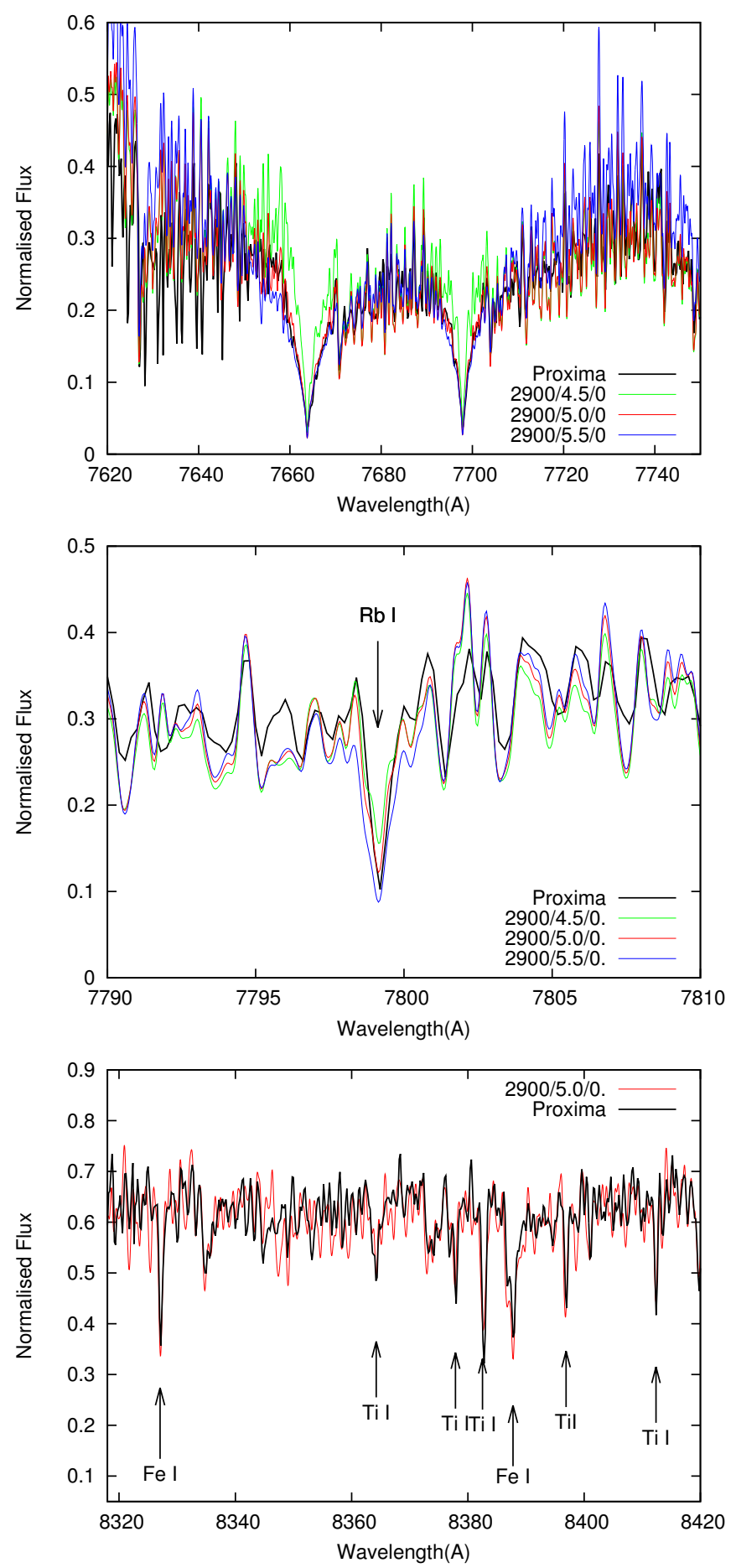
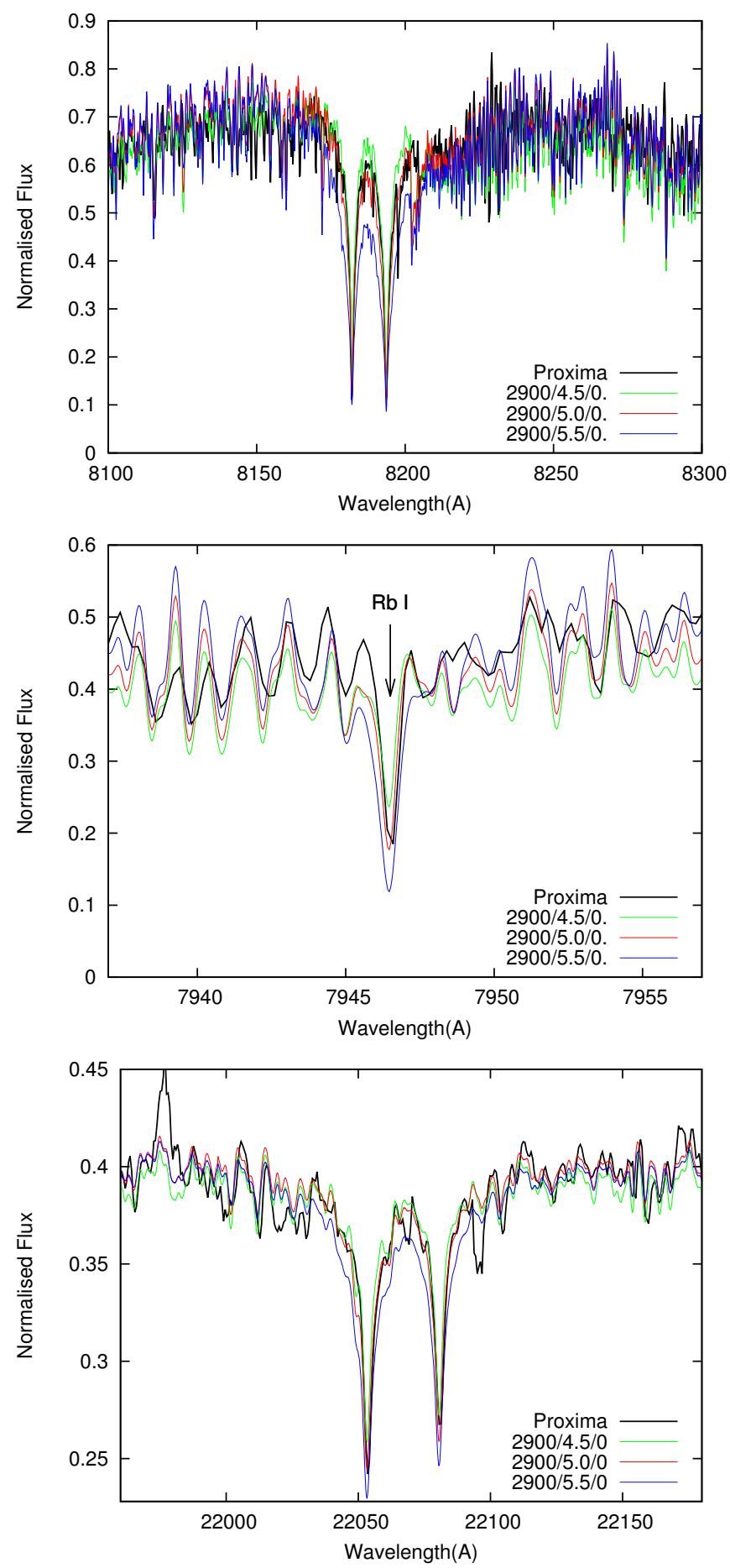

Fig. A.1. Top panel: fits to the observed KI resonance doublet (left), subordinate triplet of NaI (right). Middle panel: fits to RbI resonance doublet lines. Bottom panel: fits to the observed profiles of TiI, FeI, and NaI lines. 
Y. Pavlenko et al.: Spectrum analysis of Proxima Cen
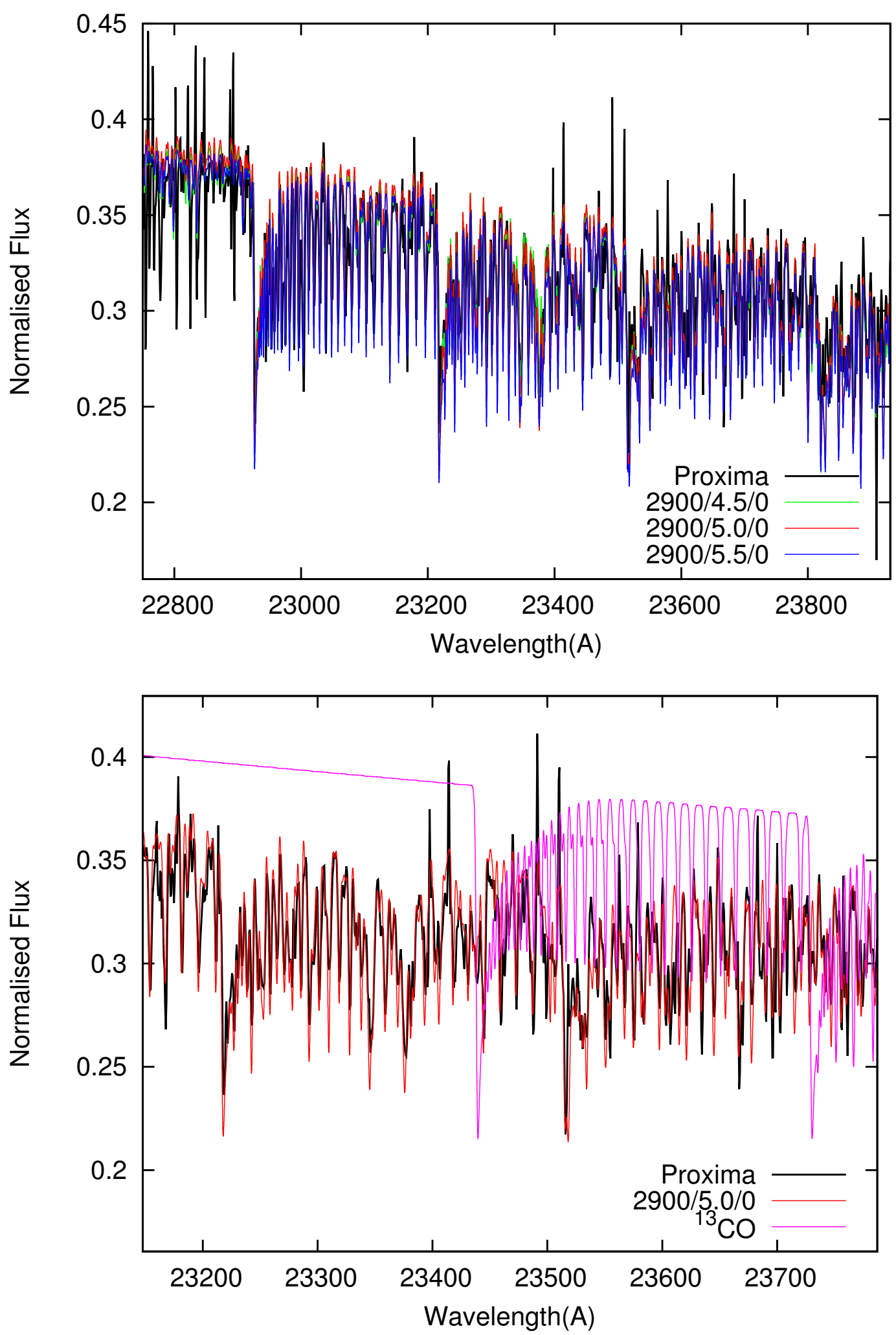

Fig. A.2. Top: fit of our synthetic spectra governed mainly by ${ }^{12} \mathrm{CO}$ bands to the observed VLT/X-shooter spectrum. Bottom: ${ }^{13} \mathrm{CO}$ bands in the theoretical spectrum computed for the parameters of Proxima. 

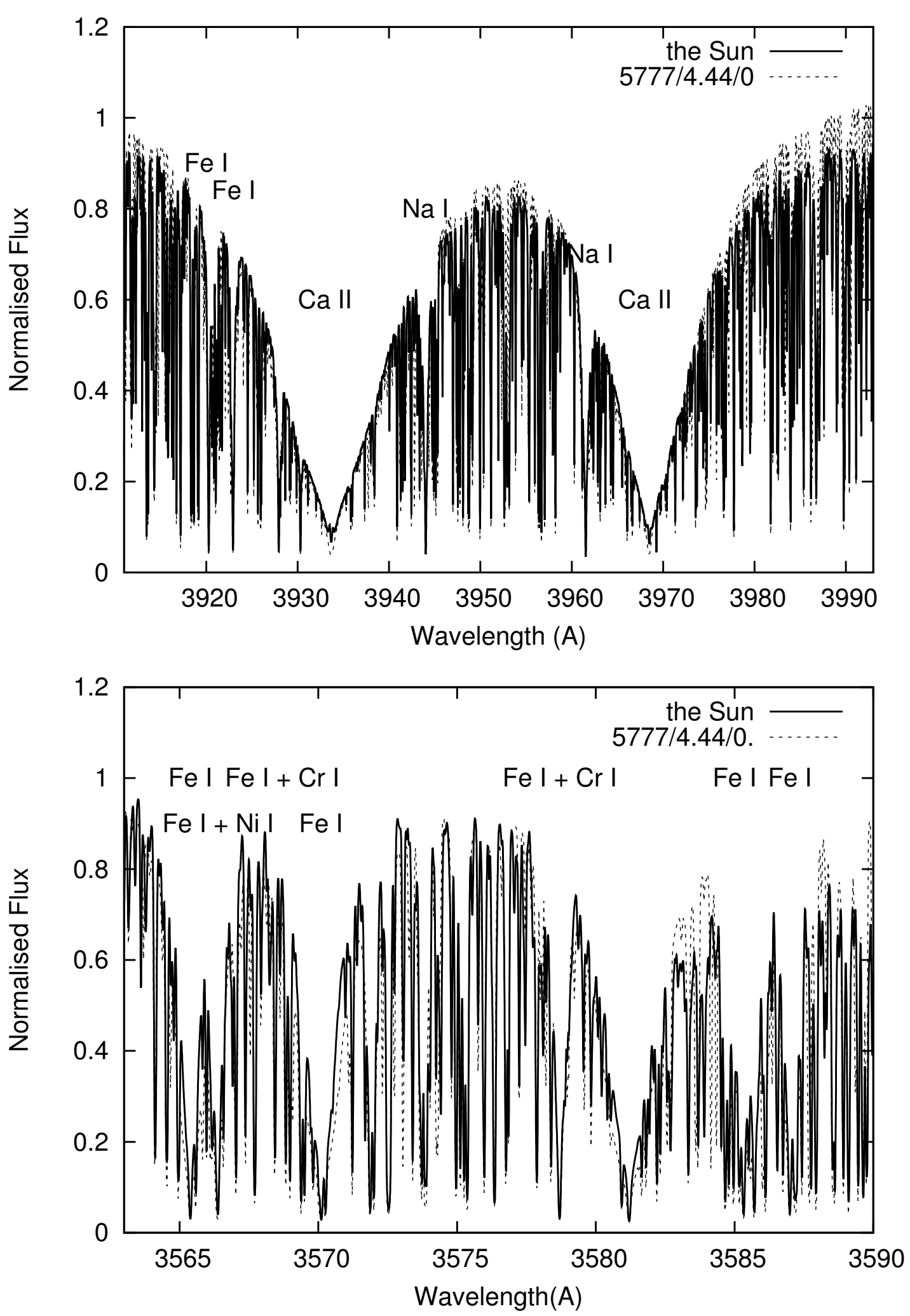

Fig. A.3. Comparison of the strong lines in the spectrum of the Sun as a star (Kurucz et al. 1984) and the theoretical spectrum computed for the 1D model atmosphere of $T_{\text {eff }} / \log g /[\mathrm{Fe} / \mathrm{H}]=5777 / 4.44 / 0.0$. 
Table A.1. Emission lines in Proxima spectrum, see Sect. 3.3.3.

\begin{tabular}{|c|c|c|c|c|c|}
\hline$\lambda$ & Element & $g f$ & $E^{\prime \prime}(\mathrm{eV})$ & & Remarks \\
\hline 3819.57 & $\mathrm{Cr} \mathrm{I}$ & $1.16 \mathrm{E}+00$ & 2.708 & $\mathrm{~S}, \mathrm{QC}$ & ecs \\
\hline 3824.44 & FeI & $4.35 \mathrm{E}-02$ & 0.0 & S, QC & ec \\
\hline 3829.36 & Mg I & $5.93 \mathrm{E}-01$ & 2.709 & $\mathrm{~S}, \mathrm{QC}$ & ec \\
\hline 3832.30 & $\mathrm{Mg} \mathrm{I}$ & $1.33 \mathrm{E}+00$ & 2.712 & S, QC & ec \\
\hline 3837.60 & & & & S, QC & \\
\hline 3838.29 & Mg I & $2.50 \mathrm{E}+00$ & 2.717 & S, QC & ec \\
\hline 3840.75 & V I & $7.28 \mathrm{E}-01$ & 0.040 & S, QC & ecs \\
\hline 3847.33 & V I & $8.51 \mathrm{E}-02$ & 0.017 & S, QC & ecs, P Cyg? \\
\hline 3853.88 & & & & S, QC & \\
\hline 3856.37 & FeI & $5.18 \mathrm{E}-02$ & 0.052 & $\mathrm{~S}, \mathrm{QC}$ & ec \\
\hline 3859.91 & FeI & $1.95 \mathrm{E}-01$ & 0.000 & $\mathrm{~S}, \mathrm{QC}$ & ec \\
\hline 3864.10 & Mo I & $9.77 \mathrm{E}-01$ & 0.000 & $S$ & ec, iP Cyg? \\
\hline 3870.91 & & & & $\mathrm{~S}>\mathrm{QC}$ & \\
\hline 3872.50 & FeI & $1.18 \mathrm{E}-01$ & 0.990 & $\mathrm{~S}, \mathrm{QC}$ & ec \\
\hline 3878.57 & FeI & 4.18E-02 & 0.087 & $\mathrm{~S}, \mathrm{QC}$ & ec \\
\hline 3886.28 & FeI & 8.39E-02 & 0.052 & S, QC & \\
\hline 3887.05 & FeI & $7.18 \mathrm{E}-02$ & 0.915 & S, QC & \\
\hline 3888.66 & $\mathrm{HeI}$ & & & S, QC & \\
\hline 3894.03 & $\mathrm{Cr} \mathrm{I}$ & 2.24E-02 & 0.961 & $\mathrm{~S}, \mathrm{QC}$ & ec \\
\hline 3894.08 & Co I & $1.26 \mathrm{E}+00$ & 1.049 & $\mathrm{~S}, \mathrm{QC}$ & ec \\
\hline 3894.98 & Co I & $3.98 \mathrm{E}-02$ & 0.629 & $\mathrm{~S}, \mathrm{QC}$ & ec \\
\hline 3895.66 & FeI & $2.14 \mathrm{E}-02$ & 0.110 & S, QC & ec \\
\hline 3897.88 & FeI & $1.84 \mathrm{E}-01$ & 2.692 & S, QC & iP Cyg \\
\hline 3899.71 & FeI & $2.94 \mathrm{E}-02$ & 0.087 & $\mathrm{~S}, \mathrm{QC}$ & ecs \\
\hline 3903.16 & $\mathrm{Cr} \mathrm{I}$ & $5.89 \mathrm{E}-03$ & 0.968 & S, QC & ec \\
\hline 3903.90 & FeI & $1.57 \mathrm{E}-01$ & 2.990 & QC & \\
\hline 3905.52 & $\mathrm{Si} \mathrm{I}$ & $9.10 \mathrm{E}-02$ & 1.909 & $\mathrm{~S}, \mathrm{QC}$ & \\
\hline 3906.48 & FeI & $5.71 \mathrm{E}-03$ & 0.110 & $\mathrm{~S}, \mathrm{QC}$ & ecs \\
\hline 3907.49 & sr I & $2.28 \mathrm{E}+00$ & 0.000 & $\mathrm{~S}, \mathrm{QC}$ & ec \\
\hline 3907.93 & FeI & 7.64E-02 & 2.759 & $\mathrm{~S}, \mathrm{QC}$ & ec \\
\hline 3908.76 & $\mathrm{Cr} \mathrm{I}$ & 8.91E-02 & 1.004 & $\mathrm{~S}$ & ec shifted to red \\
\hline 3909.86 & V I, Co I & $7.94 \mathrm{E}-02$ & 0.069 & $\mathrm{~S}, \mathrm{QC}$ & complicated blend \\
\hline 3920.26 & FeI & $1.80 \mathrm{E}-02$ & 0.121 & $\mathrm{~S}, \mathrm{QC}$ & ecs shifted to red \\
\hline 3922.91 & FeI & $2.23 \mathrm{E}-02$ & 0.052 & S, QC & \\
\hline 3926.82 & & & & $\mathrm{~S}, \mathrm{QC}$ & esc \\
\hline 3927.92 & FeI & $3.01 \mathrm{E}-02$ & 0.110 & S, QC & ec \\
\hline 3930.30 & FeI & $3.23 \mathrm{E}-02$ & 0.087 & $\mathrm{~S}, \mathrm{QC}$ & ec \\
\hline 3939.26 & & & & $S$ & iP Pyg \\
\hline 3940.88 & FeI & $2.51 \mathrm{E}-03$ & 0.958 & $\mathrm{~S}, \mathrm{QC}$ & ec shifted to blue \\
\hline 3941.49 & Cr I & 4.07E-02 & 1.030 & $\mathrm{~S}, \mathrm{QC}$ & ec shifted to blue \\
\hline 3941.73 & Co I & $9.33 \mathrm{E}-03$ & 0.432 & QC & ecs \\
\hline 3944.01 & Al I & $2.38 \mathrm{E}-01$ & 0.000 & $\mathrm{QC}, \mathrm{S}$ & ecs \\
\hline 3948.67 & TiI & $3.98 \mathrm{E}-01$ & 0.000 & $\mathrm{~S}, \mathrm{QC}$ & $\mathrm{ec}$ \\
\hline 3960.05 & & & & $\mathrm{~S}$ & \\
\hline 3961.52 & Al I & $4.75 \mathrm{E}-01$ & 0.014 & QC, S & ecs \\
\hline 3983.13 & & & & QC, S & \\
\hline 4001.66 & FeI & $1.26 \mathrm{E}-02$ & 2.176 & $\mathrm{QC}, \mathrm{S}$ & ec shifted to blue \\
\hline 4021.87 & FeI & $1.87 \mathrm{E}-01$ & 2.759 & $\mathrm{QC}, \mathrm{S}$ & ec \\
\hline 4026.19 & & & & QC, S & He II \\
\hline 4030.75 & Mn I & $3.21 \mathrm{E}-01$ & 0.0 & QC, S & ecs \\
\hline 4032.98 & $\mathrm{Ga} \mathrm{I}$ & $2.36 \mathrm{E}-01$ & 0.0 & $\mathrm{~S}$ & \\
\hline 4033.06 & Mn I & $2.27 \mathrm{E}-01$ & 0.0 & QC, S & ecs \\
\hline 4034.48 & Mn I & $1.44 \mathrm{E}-01$ & 0.0 & QC, S & $\mathrm{ecC}$ \\
\hline 4042.93 & & & & QC, S & \\
\hline 4044.14 & 19.00 & $1.20 \mathrm{E}-02$ & 0.000 & QC, S & P Cyg \\
\hline 4045.97 & Dy I & $7.08 \mathrm{E}+00$ & 0.0 & $S$ & ec \\
\hline 4047.21 & K I & $6.03 \mathrm{E}-03$ & 0.0 & QC, S & ecs \\
\hline 4052.96 & & & & $\mathrm{QC}, \mathrm{S}$ & \\
\hline
\end{tabular}


Table A.1. continued.

\begin{tabular}{|c|c|c|c|c|c|}
\hline$\lambda$ & Element & $g f$ & $E^{\prime \prime}(\mathrm{eV})$ & & Remarks \\
\hline 4062.44 & FeI & $1.37 \mathrm{E}-01$ & 2.845 & QC, S & ecs \\
\hline 4063.59 & FeI & $1.15 \mathrm{E}+00$ & 1.557 & $\mathrm{QC}$ & ecs \\
\hline 4064.21 & TiI & $1.20 \mathrm{E}-01$ & 1.053 & QC, S & ec \\
\hline 4071.74 & FeI & $9.51 \mathrm{E}-01$ & 1.608 & QC, $\mathrm{S}$ & P Cyg \\
\hline 4077.36 & Y I & $1.89 \mathrm{E}+00$ & 0.0 & QC, $\mathrm{S}$ & iP Cyg \\
\hline 4077.71 & Sr II & $1.47 \mathrm{E}+00$ & 0.0 & QC, $\mathrm{S}$ & P Cyg \\
\hline 4103.94 & & & & QC, S & \\
\hline 4111.14 & & & & na? P Cyg, ecs & \\
\hline 4115.18 & V I & $1.18 \mathrm{E}+00$ & 0.287 & $\mathrm{QC}, \mathrm{S}$ & ec \\
\hline 4116.47 & V I & 4.90E-01 & 0.275 & $\mathrm{QC}, \mathrm{S}$ ec & \\
\hline 4116.56 & V I & $1.47 \mathrm{E}-01$ & 0.262 & $\mathrm{QC}, \mathrm{S}$ & ec \\
\hline 4131.99 & V I & $8.51 \mathrm{E}-01$ & 0.287 & QC, $\mathrm{S}$ & $-30 \mathrm{~km} \mathrm{~s}^{-1}$ emission. iP Cyg. \\
\hline 4132.06 & FeI & $2.11 \mathrm{E}-01$ & 1.608 & $\mathrm{QC}, \mathrm{S}$ & the same \\
\hline 4134.48 & V I & $5.94 \mathrm{E}-01$ & 0.301 & QC, S & ecs \\
\hline $4158.62 ?$ & & & & $\mathrm{QC}, \mathrm{S}$ & O II? \\
\hline $4158.67 ?$ & & & & QC, S & O II? \\
\hline 4159.68 & V I & $1.86 \mathrm{E}-02$ & 0.287 & $\mathrm{QC}, \mathrm{S}$ & ec shifted to red \\
\hline 4164.658 & $\mathrm{Nb} \mathrm{I}$ & 7.413E-01 & 0.049 & QC, $\mathrm{S}$ & in $-30 \mathrm{~km} \mathrm{~s}^{-1}$ emission line \\
\hline 4167.270 & Gd I & $1.542 \mathrm{E}-01$ & 0.124 & QC, S & P Cyg? \\
\hline 4169.877 & $\mathrm{Ce}$ II & 4.467E-01 & 0.536 & QC,,$S$ & P Cyg? \\
\hline 4173.44 & & & & $\mathrm{QC}, \mathrm{S}$ & iP Cyg \\
\hline 4178.85 & & & & QC, S & forbidden FeI? \\
\hline 4181.93 & & & & $\mathrm{QD}, \mathrm{S}$ & \\
\hline 4184.07 & & & & QD,S & \\
\hline 4185.15 & & & & QD,S & S \\
\hline 4191.09 & & & & QD,S & S \\
\hline 4198.30 & FeI & $1.91 \mathrm{E}-01$ & 2.399 & $\mathrm{QC}>\mathrm{S}$ & P Cyg \\
\hline 4200.7 & & & & $\mathrm{QC}>\mathrm{S}$ & nebular? \\
\hline 4215.52 & Sr II & $7.16 \mathrm{E}-01$ & 0.0 & QC, S & \\
\hline 4216.18 & FeI & $4.41 \mathrm{E}-04$ & 0.0 & $\mathrm{QC}, \mathrm{S}$ & ec shifted to blue \\
\hline 4226.73 & $\mathrm{Ca} \mathrm{I}$ & $1.75 \mathrm{E}+00$ & 0.0 & QC, S & ecs shifted to the red \\
\hline 4227.43 & 26.00 & $1.84 \mathrm{E}+00$ & 3.332 & QC, $\mathrm{S}$ & P Cyg \\
\hline 4233.15 & & & & $\mathrm{QC}, \mathrm{S}$ & \\
\hline 4237.27 & & & & $\mathrm{QC}, \mathrm{S}$ & \\
\hline 4254.35 & $\mathrm{Cr} \mathrm{I}$ & $8.13 \mathrm{E}-01$ & 0.000 & QC, S & \\
\hline 4259.31 & V I & $6.76 \mathrm{E}-03$ & 0.017 & QC, S & P Cyg \\
\hline 4266.34 & & & & QC, S & \\
\hline 4272.23 & & & & QC, S & \\
\hline 4274.81 & $\mathrm{Cr} \mathrm{I}$ & $6.03 \mathrm{E}-01$ & 0.0 & $\mathrm{QC}, \mathrm{S}$ & ec \\
\hline 4277.538 & TiII & $1.816 \mathrm{E}-01$ & 4.969 & QC,,$S$ & P Cyg \\
\hline 4289.73 & $\mathrm{Cr} \mathrm{I}$ & $4.27 \mathrm{E}-01$ & 0.0 & QC, $\mathrm{S}$ & ec \\
\hline 5889.95 & $\mathrm{NaI}$ & $1.28 \mathrm{E}+00$ & 0.0 & QC, S & ecs \\
\hline 5895.92 & $\mathrm{Na}$ II & $6.40 \mathrm{E}-01$ & 0.0 & QC,, $\mathrm{S}$ & ecs \\
\hline
\end{tabular}

